\title{
Generalized quiver mutations and single-centered indices
}

\author{
Jan Manschot, ${ }^{a}$ Boris Pioline $^{b, c}$ and Ashoke Sen ${ }^{d}$ \\ ${ }^{a}$ Institut Camille Jordan, Université Claude Bernard Lyon 1, \\ 43 boulevard du 11 novembre 1918, 69622 Villeurbanne cedex, France \\ ${ }^{b}$ CERN PH-TH, \\ Case C01600, CERN, CH-1211 Geneva 23, Switzerland \\ ${ }^{c}$ Laboratoire de Physique Théorique et Hautes Energies, CNRS UMR 7589, \\ Université Pierre et Marie Curie, \\ 4 place Jussieu, 75252 Paris cedex 05, France \\ ${ }^{d}$ Harish-Chandra Research Institute, \\ Chhatnag Road, Jhusi, Allahabad 211019, India \\ E-mail: jan.manschot@univ-lyon1.fr, boris.pioline@cern.ch, \\ sen@hri.res.in
}

ABSTRACT: Quiver quantum mechanics is invariant under Seiberg duality. A mathematical consequence is that the cohomology of the Higgs branch moduli space is invariant under mutations of the quiver. The Coulomb branch formula, on the other hand, conjecturally expresses the Poincaré/Dolbeault polynomial of the Higgs branch moduli space in terms of certain quantities known as single-centered indices. In this work we determine the transformations of these single-centered indices under mutations. Moreover, we generalize these mutations to quivers whose nodes carry single-centered indices different from unity. Although the Higgs branch description of these generalized quivers is currently unknown, the Coulomb branch formula is conjectured to be invariant under generalized mutations.

Keywords: Black Holes in String Theory, Duality in Gauge Field Theories, Differential and Algebraic Geometry

ArXiv EPrint: 1309.7053 


\section{Contents}

1 Introduction and summary 1

1.1 Review of quiver invariants and the Coulomb branch formula 3

1.2 Generalized quivers and generalized mutations 4

$\begin{array}{lll}1.3 & \text { Outline } & 8\end{array}$

2 Motivation for the generalized mutation conjecture $\quad 8$

2.1 Semi-primitive Coulomb formula and Fermi flip 9

$\begin{array}{ll}2.2 & \text { Transformation rule of single-centered indices } \\ \end{array}$

$\begin{array}{lll}2.3 & \text { Dependence on the choice of FI parameters } & 12\end{array}$

3 Examples of ordinary quiver mutations $\quad 14$

$\begin{array}{lll}4 & \text { Examples of generalized quiver mutations } & 19\end{array}$

\section{Introduction and summary}

Originally introduced in order to describe D-branes at orbifold singularities [1], quiver quantum mechanics has become a powerful tool for determining the spectrum of BPS states both in four-dimensional gauge theories with $\mathcal{N}=2$ global supersymmetries [2-11] and in four-dimensional type II string vacua with the same amount of local supersymmetry [3, 12-15]. Physically, quiver quantum mechanics encodes the low energy dynamics of open strings stretched between D-brane constituents, and BPS bound states are identified as cohomology classes on the Higgs branch. Mathematically, the latter is interpreted as the moduli space of semi-stable quiver representations [16].

For quivers without oriented loops, such that the superpotential vanishes, the Higgs branch cohomology can be computed systematically [17]. Equivalently, it can be computed on the Coulomb branch, by studying the quantum mechanics of a set of point-like charged particles associated with the nodes of the quiver, and interacting by Coulomb and Lorentztype forces according to the number of arrows between any two nodes [13]. The classical moduli space of such multi-centered solutions is a finite dimensional compact symplectic space [18], and the corresponding supersymmetric quantum mechanics [19-21] can be solved using localization techniques [19, 22, 23] (see [24] for a recent review). Agreement between the two approaches for any choice of stability condition (equivalently, Fayet-Iliopoulos or FI parameters) was demonstrated recently in [23, 25].

For quivers with loops, the situation is much more involved: on the Higgs branch side, there is currently no systematic way to compute the cohomology of a quiver with generic superpotential, except for Abelian quivers which can be treated by ad hoc methods [26-29]. On the Coulomb branch side, the BPS phase space is in general no longer compact, due to 
the occurence of scaling solutions $[14,30]$ where three or more constituents approach each other at arbitrary small distance. While the symplectic volume of this phase space is still finite $[18,19]$, the number of associated Coulomb branch states fails to match the number of states on the Higgs branch, by an exponential amount [14]. Based on the observation on simple cases that the discrepancy originates solely from the middle cohomology (more precisely, the Lefschetz singlet part thereof) and is insensitive to wall-crossing [26], it was proposed in [29] that the isomorphism between the Coulomb and Higgs branch could be restored by postulating the existence of new Coulomb branch constituents, behaving as point-like particles carrying composite charge $\gamma$ and internal degrees of freedom with index $\Omega_{\mathrm{S}}(\gamma)$, insensitive to the choice of stability condition. Conjecturally, the Poincaré-Laurent polynomial of the quiver moduli space (defined in (1.2) below) is expressed in terms of these invariants, known as single-centered indices (or indices associated with pure Higgs, or intrinsic Higgs states) through the Coulomb branch formula (see (1.3)). Defining and computing the single-centered indices $\Omega_{\mathrm{S}}(\gamma)$ directly remains an open problem.

While there is no general prescription for computing the Poincaré-Laurent polynomial of a quiver with generic superpotential, it is known to be invariant under specific transformations of the quiver known as mutations [31-33]. Quiver mutation was first introduced in the context of ADE quivers [34], and is one of the basic principles of the theory of cluster algebras [35]. In terms of the quiver quantum mechanics descriptions of BPS bound states, mutations are a manifestation of Seiberg duality [36-42], and arise when the splitting between BPS and anti-BPS states is varied [9, 15, 43, 44]. This happens in particular when the moduli are varied around a point where one of the constituents of the bound state becomes massless, and is responsible for the monodromy transformation of the BPS spectrum [15, 44]. A natural question is to determine the action of mutations on the single-centered invariants $\Omega_{\mathrm{S}}(\gamma)$ appearing in the Coulomb branch formula.

From the point of view of the Coulomb branch formula, however, quiver moduli spaces are but a very special case where the basis vectors associated to the nodes of the quiver carry unit index, $\Omega_{\mathrm{S}}\left(\gamma_{i}\right)=1$ and $\Omega_{\mathrm{S}}\left(\ell \gamma_{i}\right)=0$ if $\ell>1$ (mathematically, the nodes represent spherical objects in the derived category of representations). Formally, one could very well keep the same quiver topology but associate different indices $\Omega_{\mathrm{S}}\left(\gamma_{i}\right)$ to the basis vectors and multiples thereof, and use the Coulomb branch formula to produce a set of symmetric Laurent polynomials satisfying the standard wall-crossing properties. We refer to such quivers with non-standard single-centered indices as generalized quivers, and to the corresponding Laurent polynomials as generalized quiver invariants. Ref. [19] showed that, in the case of quivers without closed loops, such generalized quivers appear in wall-crossing formulas for Donaldson-Thomas invariants [32, 45]. Whether or not the generalized quiver invariants correspond to the Poincaré/Dolbeault polynomial of a putative moduli space is unclear to us at this stage, but we can ask whether invariance under mutations can be extended to this set of polynomials. A suggestive fact is that mutations can also be defined for cluster algebras with skew-symmetrizable - as opposed to skew-symmetric exchange matrix, which are naturally represented by quivers with multiplicity [46-48].

Another reason to expect such a generalization is the physical 'Fermi flip' picture of mutation developed in the context of split attrator flows in supergravity in [44]. Namely, 
in the vicinity of certain walls in moduli space (conjugation walls in the language of [44], or walls of the second kind in the language of [32]), the representation of a BPS state of total charge $\gamma=\gamma_{j}+N \gamma_{k}$ as a halo of particles carrying charges $\ell_{i} \gamma_{k}$ with $\ell_{i}>0$ orbiting around a core of charge $\gamma_{j}$ can become invalid, and needs to be replaced by a halo of particles carrying charges $-\ell_{i} \gamma_{k}$ with $\ell_{i}>0$ around a core of charge $\gamma_{j}+M_{j} \gamma_{k}$, for some positive integer $M_{j}$ [44]. This is possible when the particles of charge $\ell \gamma_{k}$ behave as fermions (i.e. carry positive ${ }^{1}$ index), so that the Fermi vacuum can be replaced by the filled Fermi sea. In this paper, we shall argue that this picture applies just as well for generalized quivers with oriented loops, and naturally suggests that the Laurent polynomials produced by the Coulomb branch formula are invariant under a generalized mutation transformation. Before stating this transformation, we need to set up some notations.

\subsection{Review of quiver invariants and the Coulomb branch formula}

Consider a quiver with $K$ nodes with dimension vectors $\left(N_{1}, \cdots N_{K}\right)$, stability (or FayetIliopoulos, or FI) parameters $\left(\zeta_{1}, \cdots \zeta_{K}\right)$ satisfying $\sum_{i=1}^{K} N_{i} \zeta_{i}=0$, and $\gamma_{i j}$ arrows from the $i$-th node to the $j$-th node. We denote such a quiver by $\mathcal{Q}(\gamma ; \zeta)$, where $\gamma$ is a vector $\gamma=\sum_{i=1}^{K} N_{i} \gamma_{i}$ in a $K$-dimensional lattice $\Gamma$ spanned by basis vectors $\gamma_{i}$ associated to each node. We shall denote by $\Gamma^{+}$the collection of lattice vectors of the form $\sum_{i} n_{i} \gamma_{i}$ with $n_{i} \geq 0$; clearly all physical quivers are described by some vector $\gamma \in \Gamma^{+}$. We introduce a bilinear symplectic product (the Dirac-Schwinger-Zwanziger, or DSZ product) on $\Gamma$ via $\left\langle\gamma_{i}, \gamma_{j}\right\rangle=\gamma_{i j}$. To define the quiver moduli space, we introduce complex variables $\phi_{\ell k, \alpha, s s^{\prime}}$ for every pair $\ell, k$ for which $\gamma_{\ell k}>0$. Here $\alpha$ runs over $\gamma_{\ell k}$ values, $s$ is an index labelling the fundamental representation of $\mathrm{U}\left(N_{\ell}\right)$ and $s^{\prime}$ is an index representing the anti-fundamental representation of $\mathrm{U}\left(N_{k}\right)$. The moduli space $\mathcal{M}(\gamma ; \zeta)$ of classical vacua is the space of solutions to the D-term and F-term constraints,

$$
\begin{aligned}
& \sum_{\substack{k, s, t, s^{\prime} \\
\gamma_{\ell k}>0}} \phi_{\ell k, \alpha, s s^{\prime}}^{*} T_{s t}^{a} \phi_{\ell k, \alpha, t s^{\prime}}-\sum_{\substack{k, s, t, s^{\prime} \\
\gamma_{k \ell}>0}} \phi_{k \ell, \alpha, s^{\prime} s}^{*} T_{s t}^{a} \phi_{k \ell, \alpha, s^{\prime} t}=\zeta_{\ell} \operatorname{Tr}\left(T^{a}\right) \quad \forall \ell, a, \\
& \frac{\partial W}{\partial \phi_{\ell k, \alpha, s s^{\prime}}}=0,
\end{aligned}
$$

modded out by the natural action of the gauge group $\prod_{\ell} \mathrm{U}\left(N_{\ell}\right)$. Here $T^{a}$ 's are the generators of the $\mathrm{U}\left(N_{\ell}\right)$ gauge group, and $W$ is a generic gauge invariant superpotential holomorphic in the variables $\phi_{\ell k, \alpha, s s^{\prime}}$. For generic potential, $\mathcal{M}(\gamma ; \zeta)$ is a compact algebraic variety, which is smooth if the vector $\gamma$ is primitive.

Let $Q(\gamma ; \zeta ; y)$ be the Poincaré-Laurent polynomial of the quiver moduli space $\mathcal{M}(\gamma ; \zeta)$,

$$
Q(\gamma ; \zeta ; y)=\sum_{p=0}^{2 d} b_{p}(\mathcal{M})(-y)^{p-d}
$$

where $d$ is the complex dimension of $\mathcal{M}$ and the $b_{p}(\mathcal{M})$ 's are the topological Betti numbers of $\mathcal{M}$. The Coulomb branch formula for $Q(\gamma ; \zeta ; y)$, which we denote by $Q_{\text {Coulomb }}(\gamma ; \zeta ; y)$,

\footnotetext{
${ }^{1}$ Due to the supermultiplet structure a state with positive index behaves as a fermion while forming a bound state [13].
} 
takes the form $[19,23,29]$

$$
\begin{aligned}
Q_{\text {Coulomb }}(\gamma ; \zeta ; y)= & \sum_{m \mid \gamma} \frac{\mu(m)}{m} \frac{y-y^{-1}}{y^{m}-y^{-m}} \bar{Q}_{\text {Coulomb }}\left(\gamma / m ; \zeta ; y^{m}\right), \\
\bar{Q}_{\text {Coulomb }}(\gamma ; \zeta ; y)= & \sum_{n \geq 1} \sum_{\substack{\left\{\alpha_{i} \in \Gamma^{+}\right\} \\
\sum_{i=1}^{n} \alpha_{i}=\gamma}} \frac{g_{\text {Coulomb }}\left(\left\{\alpha_{1}, \cdots, \alpha_{n}\right\},\left\{c_{1}, \cdots c_{n}\right\} ; y\right)}{\left|\operatorname{Aut}\left(\left\{\alpha_{1}, \cdots, \alpha_{n}\right\}\right)\right|} \\
& \times \prod_{i=1}^{n}\left\{\sum_{\substack{m_{i} \in \mathbb{Z} \\
m_{i} \mid \alpha_{i}}} \frac{1}{m_{i}} \frac{y-y^{-1}}{y^{m_{i}}-y^{-m_{i}}} \Omega_{\text {tot }}\left(\alpha_{i} / m_{i} ; y^{m_{i}}\right)\right\},
\end{aligned}
$$

where $\mu(m)$ is the Möbius function, $\left|\operatorname{Aut}\left(\left\{\alpha_{1}, \cdots \alpha_{n}\right\}\right)\right|$ is a symmetry factor given by $\prod_{k} s_{k}$ ! if among the set $\left\{\alpha_{i}\right\}$ there are $s_{1}$ identical vectors $\tilde{\alpha}_{1}, s_{2}$ identical vectors $\tilde{\alpha}_{2}$ etc., and $m \mid \alpha$ means that $m$ is a common divisor of $\left(n_{1}, \cdots, n_{K}\right)$ if $\alpha=\sum_{\ell} n_{\ell} \gamma_{\ell}$. The sums over $n$ and $\left\{\alpha_{1}, \cdots \alpha_{n}\right\}$ in the second equation label all possible ways of expressing $\gamma$ as (unordered) sums of elements $\alpha_{i}$ of $\Gamma^{+}$. The coefficients $c_{i}$ are determined in terms of the FI parameters $\zeta_{i}$ by $c_{i}=\sum_{\ell} A_{i \ell} \zeta_{\ell}$ whenever $\alpha_{i}=\sum_{\ell} A_{i \ell} \gamma_{\ell}$. From the restrictions $\sum_{i} \alpha_{i}=\gamma$ and $\sum_{\ell} N_{\ell} \zeta_{\ell}=0$ it follows that $\sum_{i} c_{i}=0$. The functions $g_{\text {Coulomb }}\left(\left\{\alpha_{1}, \cdots, \alpha_{n}\right\} ;\left\{c_{1}, \cdots c_{n}\right\} ; y\right)$, known as Coulomb indices, can be computed from the sum over collinear solutions to Denef's equations for multi-centered black hole solutions [19]. The functions $\Omega_{\mathrm{tot}}(\alpha ; y)$ are expressed in terms of the single-centered BPS invariants $\Omega_{\mathrm{S}}$ through

$$
\Omega_{\mathrm{tot}}(\alpha ; y)=\Omega_{\mathrm{S}}(\alpha ; y)+\sum_{\substack{\left\{\beta_{i} \in \Gamma^{+}\right\},\left\{m_{i} \in \mathbb{Z}\right\} \\ m_{i} \geq 1, \sum_{i} m_{i} \beta_{i}=\alpha}} H\left(\left\{\beta_{i}\right\} ;\left\{m_{i}\right\} ; y\right) \prod_{i} \Omega_{\mathrm{S}}\left(\beta_{i} ; y^{m_{i}}\right) .
$$

The $H\left(\left\{\beta_{i}\right\} ;\left\{m_{i}\right\} ; y\right)$ are determined recursively using the minimal modification hypothesis described in [29], and $\Omega_{\mathrm{S}}(\alpha ; y)$ are expected to be $y$-independent constants for quivers with generic superpotential. A fully explicit recursive algorithm for computing the Coulomb indices $g_{\text {Coulomb }}$ and $H$-factors was given in [23].

In [29] we also proposed a formula for the Dolbeault polynomial

$$
Q(\gamma ; \zeta ; y ; t) \equiv \sum_{p, q} h^{p, q}(\mathcal{M})(-y)^{p+q-d} t^{p-q},
$$

where $h^{p, q}(\mathcal{M})$ are the Hodge numbers of $\mathcal{M}$. The formula takes the same form as (1.3), (1.4), with the only difference that $\Omega_{\mathrm{S}}$ is allowed to depend on $t$, and the arguments $y$ and $y^{m}$ inside $Q_{\text {Coulomb }}, \bar{Q}_{\text {Coulomb }}, \Omega_{\text {tot }}$ and $\Omega_{\mathrm{S}}$ are replaced by $y ; t$ and $y^{m} ; t^{m}$ respectively. ${ }^{2}$ The Coulomb indices $g_{\text {Coulomb }}$ and the functions $H$ remain unchanged and independent of $t$.

\subsection{Generalized quivers and generalized mutations}

We are now ready to state our main result. As mentioned above, the Coulomb branch formula given in eqs. (1.3), (1.4) leads to a set of symmetric Laurent polynomials satisfying the standard wall-crossing formula, for any choice of symmetric Laurent polynomials

\footnotetext{
${ }^{2}$ Eventually we drop the $y$-dependence of $\Omega_{\mathrm{S}}$ for quivers with generic superpotential.
} 
$\Omega_{\mathrm{S}}(\gamma ; y ; t)$. For ordinary quivers with a generic superpotential, the single-centered invariants satisfy

$$
\Omega_{\mathrm{S}}\left(n_{i} \gamma_{i}+n_{j} \gamma_{j} ; y ; t\right)= \begin{cases}1 & \text { if } n_{i}=1, n_{j}=0 \\ 1 & \text { if } n_{i}=0, n_{j}=1 \\ 0 & \text { otherwise }\end{cases}
$$

for any linear combination of two basis vectors $n_{i} \gamma_{i}+n_{j} \gamma_{j}$. We refer to quivers equipped with more general choices of the single-centered invariants $\Omega_{\mathrm{S}}(\gamma ; y ; t)$, subject to the condition that they vanish unless $\gamma \in \Gamma^{+}$, as 'generalized quivers'.

For such a generalized quiver, we introduce a generalized mutation $\mu_{k}^{\varepsilon}$ (where $\varepsilon=1$ for a right mutation, and $\varepsilon=-1$ for a left mutation) with respect to the $k$-th node, through the following transformation rules of the basis vectors $\gamma_{i}$, DSZ matrix $\gamma_{i j}$, stability parameters $\zeta_{i}$, and dimension vector $N_{i}$ :

$$
\begin{aligned}
\gamma_{i}^{\prime} & = \begin{cases}-\gamma_{k} & \text { if } i=k \\
\gamma_{i}+M \max \left(0, \varepsilon \gamma_{i k}\right) \gamma_{k} & \text { if } i \neq k\end{cases} \\
\gamma_{i j}^{\prime} & = \begin{cases}-\gamma_{i j} & \text { if } i=k \text { or } j=k \\
\gamma_{i j}+M \max \left(0, \gamma_{i k} \gamma_{k j}\right) \operatorname{sign}\left(\gamma_{k j}\right) & \text { if } i, j \neq k\end{cases} \\
\zeta_{i}^{\prime} & = \begin{cases}-\zeta_{k} & \text { if } i=k \\
\zeta_{i}+M \max \left(0, \varepsilon \gamma_{i k}\right) \zeta_{k} & \text { for } i \neq k,\end{cases} \\
N_{i}^{\prime} & = \begin{cases}-N_{k}+M \sum_{j \neq k} N_{j} \max \left(0, \varepsilon \gamma_{j k}\right) & \text { if } i=k \\
N_{i} & \text { if } i \neq k\end{cases}
\end{aligned}
$$

where $M$ is an integer defined by

$$
M \equiv \sum_{\ell \geq 1} \sum_{n, s} \ell^{2} \Omega_{n, s}\left(\ell \gamma_{k}\right), \quad \Omega_{\mathrm{S}}\left(\ell \gamma_{k} ; y ; t\right)=\sum_{n, s} \Omega_{n, s}\left(\ell \gamma_{k}\right) y^{n} t^{s}
$$

These transformation laws guarantee that

$$
\gamma \equiv \sum_{i} N_{i} \gamma_{i}=\sum_{i} N_{i}^{\prime} \gamma_{i}^{\prime}
$$

We conjecture that the Laurent polynomials produced by the Coulomb branch formula are invariant under the generalized mutation transformation: ${ }^{3}$

$$
Q_{\text {Coulomb }}(\gamma ; \zeta ; y ; t)= \begin{cases}Q_{\text {Coulomb }}^{\prime}\left(\gamma ; \zeta^{\prime} ; y ; t\right) & \text { if } \gamma \nVdash \gamma_{k} \\ Q_{\text {Coulomb }}^{\prime}(-\gamma ; \zeta ; y ; t) & \text { if } \gamma \| \gamma_{k},\end{cases}
$$

under the conditions that

\footnotetext{
${ }^{3}$ The second equation in (1.10) may be surprising at first, but physically it reflects the fact that in the transformed quiver states with charge vectors $\ell \gamma_{k}$ are considered as anti-BPS states and are no longer counted in the BPS index. On the other hand states with charge vector $-\ell \gamma_{k}$, which are considered anti-BPS in the original quiver and not counted, are taken to be BPS in the new quiver.
} 
i) $\Omega_{n, s}\left(\ell \gamma_{k}\right)$ are positive integers satisfying $\Omega_{n, s}\left(\ell \gamma_{k}\right)=\Omega_{-n,-s}\left(\ell \gamma_{k}\right)$ and vanish for $\ell$ large enough, so that the integer $M$ is well defined,

$$
\Omega_{n, s}\left(\ell \gamma_{k}\right) \geq 0 \forall \ell>0, \quad \Omega_{n, s}\left(\ell \gamma_{k}\right)=0 \text { for } \ell>\ell_{\operatorname{Max}}
$$

ii) the stability parameter $\zeta_{k}$ has sign $-\varepsilon$,

$$
\varepsilon \zeta_{k}<0
$$

iii) the single-centered indices transform as ${ }^{4}$

$$
\Omega_{\mathrm{S}}(\alpha ; y ; t)=\left\{\begin{array}{ll}
\Omega_{\mathrm{S}}^{\prime}\left(\alpha+M \max \left(0, \varepsilon\left\langle\alpha, \gamma_{k}\right\rangle\right) \gamma_{k} ; y ; t\right) & \text { for } \alpha \nVdash \gamma_{k} \\
\Omega_{\mathrm{S}}^{\prime}(-\alpha) & \text { for } \alpha \| \gamma_{k}
\end{array} .\right.
$$

In (1.10), it is understood that in computing the l.h.s. we have to express $\gamma$ as $\sum_{i} N_{i} \gamma_{i}$ treating $\gamma_{i}$ 's as the basis vectors and apply the Coulomb branch formula (1.3), (1.4) while in computing the r.h.s. we have to express $\gamma$ as $\sum_{i} N_{i}^{\prime} \gamma_{i}^{\prime}$ treating $\gamma_{i}^{\prime}$ 's as the basis vectors and then apply the Coulomb branch formula (1.3), (1.4). Since the left and right mutations $\mu_{k}^{ \pm}$are inverses of each other, we shall restrict our attention to right mutations only and set

$$
\varepsilon=1
$$

henceforth.

Several remarks about our generalized mutation conjecture are in order:

1. For ordinary quivers, $\Omega_{\mathrm{S}}\left(\ell \gamma_{k}\right)=\delta_{\ell, 1}$, hence $M=1$ and the above relations reduce to mutations of ordinary quivers with superpotential (the action on the superpotential can be found in [31]).

2. For quivers obtained from cluster algebras with skew-symmetrizable exchange matrix (i.e. a integer matrix $\hat{\gamma}_{i j}$ such that $\gamma_{i j} \equiv \hat{\gamma}_{i j} / d_{j}$ is antisymmetric for some positive integers $d_{i}$ ), the action on $\gamma_{i j}$ coincides with the mutation rule specified in [46, 47] for $M=d_{k}$.

3. Mutation invariance in general imposes additional restrictions on the single-centered invariants $\Omega_{\mathrm{S}}(\gamma ; y ; t)$, beyond the vanishing of $\Omega_{\mathrm{S}}(\gamma ; y ; t)$ for $\gamma \notin \Gamma^{+}$with respect to the original quiver. Indeed, if we denote by $\Gamma^{\prime+}$ the set of vectors $\gamma=\sum_{i} n_{i}^{\prime} \gamma_{i}^{\prime} \in \Gamma$ where all $n_{i}^{\prime}$ are non-negative, then the transformation rule (1.13) requires that $\Omega_{\mathrm{S}}(\alpha ; y ; t)$ should vanish if the mutated vector $\alpha^{\prime} \equiv \alpha+M \max \left(0,\left\langle\alpha, \gamma_{k}\right\rangle\right) \gamma_{k}$ does not lie in $\Gamma^{\prime+}$, even if $\alpha \in \Gamma^{+}$(excluding the case $\left.\alpha \| \gamma_{k}\right) .{ }^{5}$ Similarly, $\Omega_{\mathrm{S}}^{\prime}\left(\alpha^{\prime}\right)$ should vanish if $\alpha^{\prime} \in \Gamma^{\prime+}$ but $\alpha \notin \Gamma^{+}$. Another consequence of the generalized mutation symmetry is that $\Omega_{\mathrm{S}}\left(\gamma_{j}+\ell \gamma_{k}\right)$ must vanish for all $\ell \neq 0$. Indeed, for

\footnotetext{
${ }^{4}$ It is easy to verify that the rational invariants $\bar{Q}_{\text {Coulomb }}$ and $\bar{\Omega}_{\mathrm{S}}$ satisfy the same mutation transformation rules as $Q_{\text {Coulomb }}$ and $\Omega_{\mathrm{S}}$ respectively.

${ }^{5}$ The same reasoning applies to the Dolbeault-Poincaré polynomial: $Q_{\text {Coulomb }}(\gamma)=0$ if $\gamma \notin \Gamma_{+}$or $\gamma \notin \Gamma_{+}^{\prime}$.
} 
negative $\ell$, the vector $\alpha=\gamma_{j}+\ell \gamma_{k}$ fails to lie in $\Gamma^{+}$, while for positive $\ell$, the mutated vector $\alpha^{\prime}=\gamma_{j}+M \max \left(\gamma_{j k}, 0\right) \gamma_{k}+\ell \gamma_{k}=\gamma_{j}^{\prime}-\ell \gamma_{k}^{\prime}$ fails to lie in $\Gamma^{\prime+}$. If the $\Omega_{s}$ 's fail to satisfy these constraints, they still define a generalized quiver but generalized mutation symmetry does not apply. Indeed it is unclear a priori if there exists a set of single-centered invariants $\Omega_{\mathrm{S}}(\gamma ; y ; t)$ which is consistent with the above constraints arising from arbitrary sequences of mutations. Finding a Higgs branch-type realization of such generalized quivers invariant under mutations would allow to give an affirmative answer to this question.

4. A useful way to state the property (1.10) is to construct the generating functions

$$
\begin{aligned}
\mathcal{F}(\vec{N} ; \zeta ; q ; y ; t) & \equiv \sum_{N_{k}} Q_{\text {Coulomb }}\left(\sum_{i \neq k} N_{i} \gamma_{i}+N_{k} \gamma_{k} ; \zeta ; y ; t\right) q^{N_{k}}, \\
\mathcal{F}^{\prime}\left(\vec{N} ; \zeta^{\prime} ; q ; y ; t\right) & \equiv \sum_{N_{k}} Q_{\text {Coulomb }}^{\prime}\left(\sum_{i \neq k} N_{i} \gamma_{i}^{\prime}+N_{k} \gamma_{k}^{\prime} ; \zeta^{\prime} ; y ; t\right) q^{N_{k}},
\end{aligned}
$$

where, on the left-hand side, $\vec{N}$ denotes the truncated dimension vector

$$
\vec{N} \equiv\left(N_{1}, \cdots N_{k-2}, N_{k-1}, N_{k+1}, N_{k+2}, \cdots\right) .
$$

Mutation invariance for all values of $N_{k}$ is then equivalent to the functional identity

$$
\mathcal{F}(\vec{N} ; \zeta ; q ; y ; t)= \begin{cases}q^{\sum_{i \neq k} M N_{i} \max \left(\gamma_{i k}, 0\right)} \mathcal{F}^{\prime}\left(\vec{N} ; \zeta^{\prime} ; q^{-1} ; y ; t\right) & \text { for } \vec{N} \neq \overrightarrow{0} \\ \mathcal{F}^{\prime}\left(\overrightarrow{0} ; \zeta^{\prime} ; q ; y ; t\right) & \text { for } \vec{N}=\overrightarrow{0}\end{cases}
$$

We conjecture that under the assumption (1.11), both sides of this equation are in fact polynomials in $q$.

5. While the conditions i)-iii) are necessary for mutation invariance of the Dolbeault polynomials $Q_{\text {Coulomb }}(\gamma ; \zeta ; y ; t)$, it is possible to relax condition i) if one is interested only in the numerical invariants $Q_{\text {Coulomb }}(\gamma ; \zeta ; y=1 ; t=1)$. In that case we conjecture that it is sufficient that the generating function $\mathcal{F}(\vec{N} ; \zeta ; q ; 1 ; 1)$ be a polynomial in $q$, invariant under $q \rightarrow 1 / q$ (up to an overall power $q^{\sum_{j \neq k} M \max \left(\gamma_{j k}, 0\right)}$ ). This allows some of the $\Omega_{\mathrm{S}}\left(\ell \gamma_{k} ; 1 ; 1\right)$ 's to be negative. For example, for the generalized Kronecker quiver (example 1 in section 4 ), one may take $\Omega_{\mathrm{S}}\left(\gamma_{k} ; 1 ; 1\right)=-1, \Omega_{\mathrm{S}}\left(2 \gamma_{k} ; 1 ; 1\right)=1$, and $\Omega_{\mathrm{S}}\left(\ell \gamma_{k} ; 1 ; 1\right)=0$ for all other $\ell$. Then the generalized mutation $\mu_{2}^{+}$has $M=3$ and preserves the numerical invariants $Q(\gamma ; \zeta ; 1 ; 1)$. Example $2(\mathrm{~g})$ of section 4 gives another example of this phenomenon for a three-node quiver.

Although we do not have a general proof that the Coulomb branch formula is indeed invariant under such generalized mutations, we shall check it in many examples of ordinary and generalized quivers, with or without oriented loop. In some cases, mutation invariance allows to determine the complete set of single-centered indices. Another useful property of mutations is that in special cases they can reduce the total rank of the quiver, which typically reduces considerably the computation time of the Coulomb branch formula. 


\subsection{Outline}

The rest of the paper is organised as follows. In section 2 we describe the physical origin of the generalized mutation transformation rules, the transformation properties of singlecentered indices under generalized mutation and the choice of FI parameters given in (1.12). In section 3 we test the ordinary mutation symmetry of the Coulomb branch formula through several examples. In section 4 we repeat this exercise for generalized mutations.

\section{Motivation for the generalized mutation conjecture}

As mentioned in the introduction, quiver quantum mechanics describes the dynamics of open strings stretched between the various BPS constituents of a given bound state. In particular, it depends on a choice of half-space $\mathcal{H}$ in the central charge plane, such that all states whose central charge lie in $\mathcal{H}$ are deemed to be BPS, while those in the opposite half-plane are anti-BPS. As the choice of $\mathcal{H}$ is varied, it may happen that one of the constituents, with charge $\gamma_{k}$, crosses the boundary of $\mathcal{H}$ and falls on the anti-BPS side, while its CPT-conjugate with charge $-\gamma_{k}$ enters the BPS side. ${ }^{6}$ Equivalently, this may take place for a fixed choice of $\mathcal{H}$ under a variation of the asymptotic moduli (staying away from walls of marginal stability). Such a wall is sometimes known as a wall of second kind [32], or as a conjugation wall [44]. Such walls are encountered in particular when varying the moduli around a point where the central charge associated to one of the BPS constituents vanishes, see figure 1 for an example which can serve as a guidance for the discussion below.

Clearly, as the state with charge $-\gamma_{k}$ enters the BPS half-space, it cannot be viewed as a bound state of the BPS constituents with charges $\gamma_{i}$, and must therefore be considered as elementary. Consequently the vector $-\gamma_{k}$ must be taken as a new basis vector, and the other basis vectors must be changed as well so that the charges carried by the BPS states can be expressed as positive linear combinations of the basis vectors. Invariance under mutation is the statement that the same BPS states can be described either as bound states of the original BPS constituents with charge $\gamma_{i}$, or of the new BPS constituents with charge $\gamma_{i}^{\prime}$.

For this equivalence to hold, it is not necessary that the indices associated with the constituents satisfy the constraint (1.6) - indeed this constraint is generically not obeyed for bound states in gauge theory [9, section 3.2] and in supergravity (such as in the D6-D0 system, studied in more detail in [22, appendix B]). Instead, we shall allow the indices $\Omega_{\mathrm{S}}\left(\gamma_{j}\right)$ of the BPS constituents to be arbitrary symmetric Laurent polynomials in twoparameter $y$ and $t$, with support on non-negative dimension vectors $\gamma \in \Gamma^{+}$. We refer to the polynomials $Q_{\text {Coulomb }}(\gamma ; \zeta ; y ; t)$ produced by the Coulomb branch formula (1.3) as generalized quiver invariants. We also assume that $\Omega_{\mathrm{S}}\left(\gamma_{j}+\ell \gamma_{k}\right)$ vanishes for $\ell \geq 1$, and that the integers $\Omega_{n, s}\left(\ell \gamma_{k}\right)$ defined through (1.8) are all positive and vanish for some large enough $\ell$. The necessity of the first condition was discussed in the last but one paragraph of section 1.2, whereas the necessity of the second condition will become clear below. Figure 1 is an example of generalized quivers, associated to a rank 2 cluster algebra with

\footnotetext{
${ }^{6}$ We assume that the spectrum is such that no other BPS state crosses the boundary of $\mathcal{H}$ at the same time.
} 


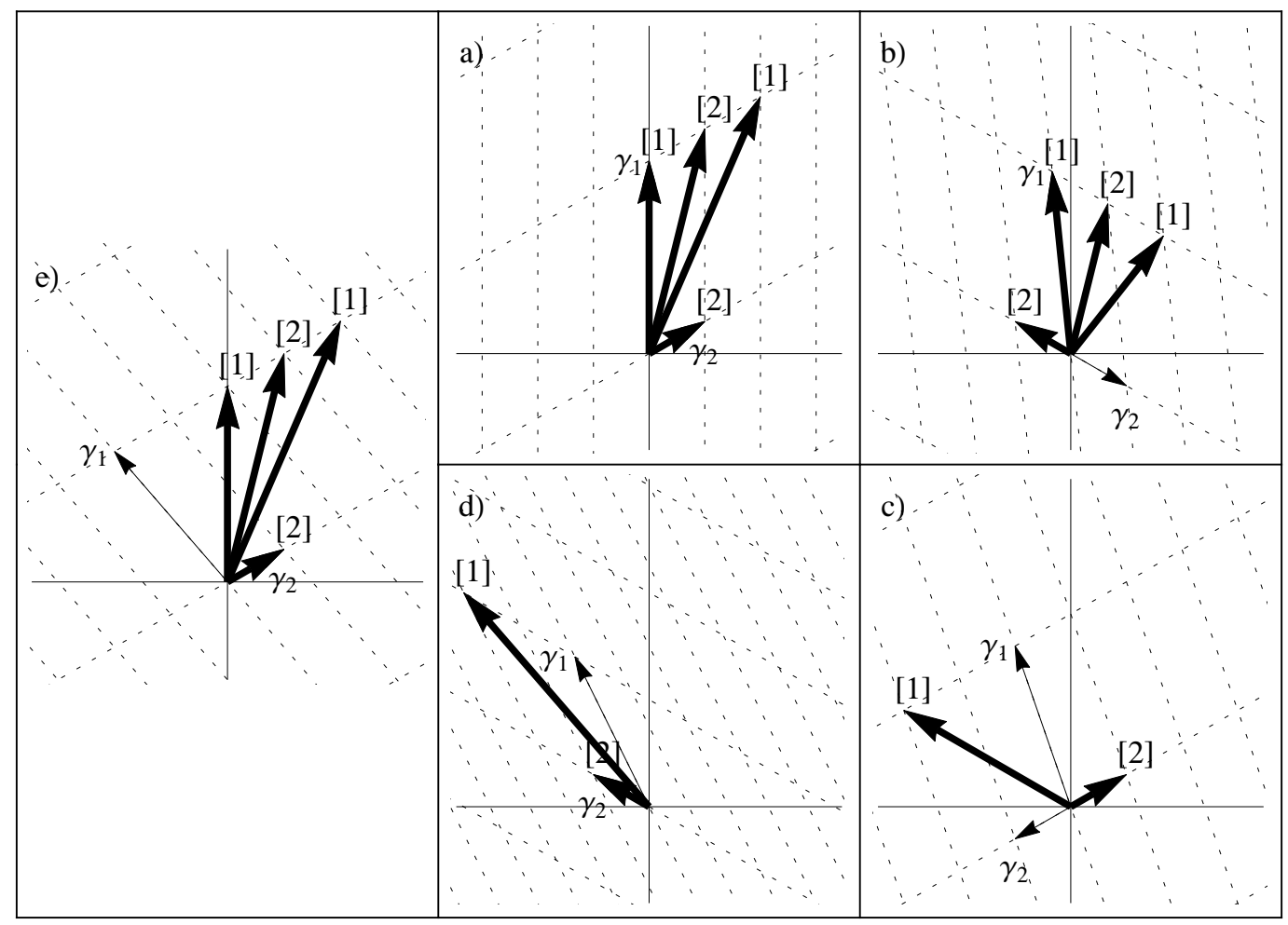

Figure 1. Spectrum of a generalized Kronecker quiver with $\gamma_{12}=1, \Omega_{\mathrm{S}}\left(\gamma_{1}\right)=1, \Omega_{\mathrm{S}}\left(\gamma_{2}\right)=2$ as the central charge $Z\left(\gamma_{2}\right)=\rho e^{i \theta}$ rotates clockwise around 0 , keeping $0<\rho \ll 1$ and $Z\left(\gamma_{1}\right)+\frac{1}{\pi}\left(\frac{\pi}{6}-\theta\right) Z\left(\gamma_{2}\right)=e^{i \pi / 2}$ fixed. The BPS half-space $\operatorname{Im}(Z)>0$ is kept fixed during the deformation. Occupied charges are depicted by an arrow in the central charge plane, decorated with the corresponding BPS index in square bracket. A conjugation wall is crossed in going from a) to b) and c) to d), while walls of marginal stability are crossed in going from b) to c) and d) to e). The spectrum in e) is identical to the spectrum in a), up to a monodromy $\gamma_{1} \mapsto \gamma_{1}+2 \gamma_{2}$. In more detail: a) $0<\theta<\pi / 2$ : the spectrum consists of 4 occupied charges $\left(\gamma_{1}, \gamma_{1}+\gamma_{2}, \gamma_{1}+2 \gamma_{2}, \gamma_{2}\right)$ and BPS indices $(1,2,1,2)$, respectively. b) $-\pi / 2<\theta<0: \gamma_{2}$ is now anti-BPS. The spectrum of the mutated quiver consists of 4 occupied charges $\left(-\gamma_{2}, \gamma_{1}, \gamma_{1}+\gamma_{2}, \gamma_{1}+2 \gamma_{2}\right)$ and indices $(2,1,2,1)$. c) $-\pi<\theta<-\pi / 2$ : the phases of the two charges $\left(\gamma_{1}+2 \gamma_{2},-\gamma_{2}\right)$ swap and they no longer form any BPS bound state. $\left.\mathrm{d}\right)$ $-3 \pi / 2<\theta<-\pi: \gamma_{2}$ re-enters the BPS half space and the spectrum of the twice-mutated quiver contains two occupied charges $\left(\gamma_{2}, \gamma_{1}+2 \gamma_{2}\right)$ with index $(2,1)$ and no bound state. e) $-2 \pi<\theta<-3 \pi / 2$, the phases of the two charges $\left(\gamma_{2}, \gamma_{1}+2 \gamma_{2}\right)$ swap again and the spectrum of the twice-mutated quiver consists of 4 occupied charges $\left(\gamma_{1}+2 \gamma_{2}, \gamma_{1}+3 \gamma_{2}, \gamma_{1}+4 \gamma_{2}, \gamma_{2}\right)$ with indices $(1,2,1,2)$.

non-symmetrizable exchange matrix with Dynkin diagram $B_{2}$ (see [49] for a similar example with Dynkin diagram $G_{2}$ ). In the rest of this section we shall describe the motivation behind the generalized mutation conjecture (1.7)-(1.13) for the generalized quiver invariants.

\subsection{Semi-primitive Coulomb formula and Fermi flip}

In order to motivate the action of mutations on the basis of BPS states, we shall focus on dimension vectors $\gamma=\gamma_{j}+N \gamma_{k}$ with support only on two nodes, the mutating node $k$ and any adjacent node $j$, hence effectively dealing with a Kronecker quiver with $\gamma_{j k}$ arrows and dimension vector $(1, N)$. 
Due to our assumption that $\Omega_{\mathrm{S}}\left(\gamma_{j}+\ell \gamma_{k}\right)=0$ for non-zero $\ell$, states carrying charge $\gamma_{j}+N \gamma_{k}$ can only arise in the original quiver as bound states of a center of charge $\gamma_{j}$ with other centers carrying charges $\ell_{i} \gamma_{k}$ with $\ell_{i}>0$. Assuming $\zeta_{k}<0<\zeta_{j}$, these states exist whenever $\gamma_{j k}>0$, and arise physically as halos of particles of charge $\ell \gamma_{k}$ orbiting around a core of charge $\gamma_{j}$ [14]. Their indices are given by the semi-primitive Coulomb branch formula [14, 22, 50],

$$
\begin{aligned}
Z & =\sum_{N} Q_{\text {Coulomb }}\left(\gamma_{j}+N \gamma_{k} ; \zeta ; y ; t\right) q^{N} \\
& =\Omega_{\mathrm{S}}\left(\gamma_{j} ; y ; t\right) \prod_{\ell \geq 1} \prod_{J=1}^{\ell \gamma_{j k}} \prod_{n} \prod_{s}\left(1+q^{\ell} t^{s} y^{n}(-y)^{2 J-\ell \gamma_{j k}-1}\right)^{\Omega_{n, s}\left(\ell \gamma_{k}\right)} .
\end{aligned}
$$

This implies that only a finite number of charge vectors $\gamma_{j}+N \gamma_{k}$ have non-zero index, namely those with $0 \leq N \leq M \gamma_{j k}$ where

$$
M \equiv \sum_{\ell=1}^{\ell_{\operatorname{Max}}} \sum_{n, s} \ell^{2} \Omega_{n, s}\left(\ell \gamma_{k}\right) .
$$

Physically $Q_{\text {Coulomb }}\left(\gamma_{j}+N \gamma_{k} ; \zeta ; y ; t\right)$ can be interpreted as the number of states corresponding to the excitations of the fermionic oscillators of charges $\ell_{i} \gamma_{k}$ in (2.1) acting on the fermionic vacuum with charge $\gamma_{j}$. As pointed out in [44], the same multiplet of states can be obtained from the filled Fermi sea of charge $\gamma_{j}^{\prime}=\gamma_{j}+M \gamma_{j k} \gamma_{k}$ by acting with fermionic oscillators of charges $\ell_{i} \gamma_{k}^{\prime}=-\ell_{i} \gamma_{k}$, provided they carry the same indices

$$
\Omega_{n, s}^{\prime}\left(\ell \gamma_{k}^{\prime}\right)=\Omega_{n, s}\left(\ell \gamma_{k}\right), \quad \Omega_{\mathrm{S}}^{\prime}\left(\gamma_{j}^{\prime} ; y ; t\right)=\Omega_{\mathrm{S}}\left(\gamma_{j} ; y ; t\right) .
$$

The particles of charge $\ell \gamma_{k}^{\prime}$ and $\gamma_{j}^{\prime}$ and the corresponding indices can be associated to the nodes of a new (generalized) quiver. In this alternative description, the bound states with charge $\gamma_{j}+N \gamma_{k}=\gamma_{j}^{\prime}+\left(M \gamma_{j k}-N\right) \gamma_{k}^{\prime}$ are described in terms of a halo of particles of charges $\ell_{i} \gamma_{k}^{\prime}$ orbiting around a core of charge $\gamma_{j}^{\prime}$. To see the equivalence of the two descriptions, one can start from the halo partition function

$$
\begin{aligned}
Z^{\prime} & \equiv \sum_{N} Q_{\text {Coulomb }}^{\prime}\left(\gamma_{j}^{\prime}+\left(M \gamma_{j k}-N\right) \gamma_{k}^{\prime} ; \zeta^{\prime} ; y ; t\right) q^{N} \\
& =q^{M \gamma_{j k}} \sum_{N^{\prime}} Q_{\text {Coulomb }}^{\prime}\left(\gamma_{j}^{\prime}+N^{\prime} \gamma_{k}^{\prime} ; \zeta^{\prime} ; y ; t\right) q^{-N^{\prime}} \\
& =q^{M \gamma_{j k}} \Omega_{\mathrm{S}}^{\prime}\left(\gamma_{j}^{\prime} ; y ; t\right) \prod_{\ell \geq 1} \prod_{J=1}^{\ell \gamma_{j k}} \prod_{n} \prod_{s}\left(1+q^{-\ell} t^{s} y^{n}(-y)^{2 J-\ell \gamma_{j k}-1}\right)^{\Omega_{n, s}^{\prime}\left(\ell \gamma_{k}^{\prime}\right)},
\end{aligned}
$$

where we have used the fact that $\gamma_{j k}^{\prime}=-\gamma_{j k}<0$ and $\zeta_{k}^{\prime}>0$. Taking out the factor of $q^{-\ell} t^{s} y^{n}(-y)^{2 J-\ell \gamma_{j k}-1}$ from each term inside the product in (2.4), using (2.3) and making a change of variable $J \rightarrow \ell \gamma_{j k}-J+1$, this can be rewritten as

$$
\begin{aligned}
Z^{\prime}= & q^{M \gamma_{j k}-\gamma_{j k} \sum_{\ell, n, s} \ell^{2} \Omega_{n, s}\left(\ell \gamma_{k}\right)} t^{\gamma_{j k} \sum_{\ell, n, s} \ell s \Omega_{n, s}\left(\ell \gamma_{k}\right)} y^{\gamma_{j k} \sum_{\ell, n, s} \ell n \Omega_{n, s}\left(\ell \gamma_{k}\right)} \\
& \times \Omega_{\mathrm{S}}\left(\gamma_{j} ; y ; t\right) \prod_{\ell \geq 1} \prod_{J=1}^{\ell \gamma_{j k}} \prod_{n} \prod_{s}\left(1+q^{\ell} t^{-s} y^{-n}(-y)^{2 J-\ell \gamma_{j k}-1}\right)^{\Omega_{n, s}\left(\ell \gamma_{k}\right)} .
\end{aligned}
$$


The exponent of $q$ in the first factor on the right hand side vanishes due to (2.2), while the exponents of $t$ and $y$ in the second and third factors vanish due to the Hodge duality symmetry $\Omega_{n, s}\left(\ell \gamma_{k}\right)=\Omega_{-n,-s}\left(\ell \gamma_{k}\right)$. The same symmetry allows us to replace the $t^{-s} y^{-n}$ term inside the product by $t^{s} y^{n}$. Thus we arrive at

$$
Z^{\prime}=\Omega_{\mathrm{S}}\left(\gamma_{j} ; y ; t\right) \prod_{\ell \geq 1}^{\ell \gamma_{j k}} \prod_{J=1} \prod_{n} \prod_{s}\left(1+q^{\ell} t^{s} y^{n}(-y)^{2 J-\ell \gamma_{j k}-1}\right)^{\Omega_{n, s}\left(\ell \gamma_{k}\right)}
$$

reproducing (2.1) whenever $\gamma_{j k}>0$. If instead $\gamma_{j k}<0$ (keeping $\zeta_{k}<0<\zeta_{j}$ ) then the first quiver does not carry any bound state of the center carrying charge $\gamma_{j}$ with centers carrying charges $\ell_{i} \gamma_{k}$ with $\ell_{i}>0$. Thus $Q_{\text {Coulomb }}\left(\gamma_{j}+N \gamma_{k}\right)$ vanishes for $N>0$. The mutated quiver describing centers of charges $\gamma_{j}^{\prime}=\gamma_{j}$ and $\ell_{i} \gamma_{k}^{\prime}=-\ell_{i} \gamma_{k}$, with indices $\Omega_{\mathrm{S}}\left(\gamma_{j} ; y ; t\right)$ and $\Omega_{\mathrm{S}}\left(\ell_{i} \gamma_{k} ; y ; t\right)$ respectively, has $\gamma_{j k}^{\prime}>0, \zeta_{j}^{\prime}<0<\zeta_{k}^{\prime}$, and therefore also no bound states of charge $\gamma_{j}^{\prime}+N \gamma_{k}^{\prime}$ for $N>0$. The partition functions $Z=Z^{\prime}=\Omega_{\mathrm{S}}\left(\gamma_{j} ; y ; t\right)$ are therefore again the same on both sides.

This shows that, under the assumptions $\zeta_{k}<0<\zeta_{j}$ and (1.11), the semi-primitive Coulomb branch formula is invariant under the transformation

$$
\begin{aligned}
& \gamma_{k}^{\prime}=-\gamma_{k}, \\
& \gamma_{j}^{\prime}=\gamma_{j}+M \max \left(0, \gamma_{j k}\right) \gamma_{k} \\
& \Omega_{\mathrm{S}}\left(\gamma_{j}\right)=\Omega_{\mathrm{S}}^{\prime}\left(\gamma_{j}^{\prime}\right), \\
& \Omega_{\mathrm{S}}^{\prime}\left(\ell \gamma_{k}^{\prime} ; y ; t\right)=\Omega_{\mathrm{S}}\left(\ell \gamma_{k} ; y ; t\right) \\
& \text { for } j \neq k \text {, } \\
& \forall \ell \text {. }
\end{aligned}
$$

This is a special case of the generalized mutation rules (1.7)-(1.13), providing the initial motivation for the conjectured invariance under the generalized mutation transformation. In the next subsections, we comment on aspects of the generalized mutation rules which are not obvious consequences of the semi-primitive case.

\subsection{Transformation rule of single-centered indices}

Let us now comment on the transformation rule (1.13) of $\Omega_{\mathrm{S}}(\alpha)$. The first equation for $\alpha=\gamma_{j}$ as well as the second equation follow from the analysis of the Kronecker quiver given above, ${ }^{7}$ but we shall now justify why this is needed for general $\alpha$. Consider two generalized quivers which are identical in all respects except that for some specific charge vector $\alpha$, the first quiver has $\Omega_{\mathrm{S}}(\alpha)=0$ while the second quiver has some non-zero $\Omega_{\mathrm{S}}(\alpha ; y ; t)$. Let us denote by $Q(\gamma)$ and $\hat{Q}(\gamma)$ the Coulomb branch formulæ for these two quivers. Now consider the difference $\hat{Q}\left(\alpha+\ell \gamma_{k}\right)-Q\left(\alpha+\ell \gamma_{k}\right)$ for some positive integer $\ell$. This difference must come from a bound state configuration of a center of charge $\alpha$ with a set of centers carrying charges parallel to $\gamma_{k}$. The index associated with this configuration is encoded in the partition function $Z$ given in (2.1) with $\gamma_{j}$ replaced by $\alpha$. Now consider the mutated version of both quivers with respect to the $k$-th node. The difference $\hat{Q}^{\prime}\left(\alpha+\ell \gamma_{k}\right)-Q^{\prime}\left(\alpha+\ell \gamma_{k}\right)$ must agree with $\hat{Q}\left(\alpha+\ell \gamma_{k}\right)-Q\left(\alpha+\ell \gamma_{k}\right)$. Our previous analysis showing the equality of $Z$ and $Z^{\prime}$ guarantees that this is achieved if we assume that the mutated quivers are identical except for one change: $\Omega_{\mathrm{S}}^{\prime}\left(\alpha+M \max \left(0,\left\langle\alpha, \gamma_{k}\right\rangle\right) \gamma_{k} ; y ; t\right)$ is zero in the first mutated quiver but is equal to $\Omega_{\mathrm{S}}(\alpha ; y ; t)$ for the second mutated quiver.

\footnotetext{
${ }^{7}$ While this paper was in preparation, this observation was also made in ref. [9].
} 
The extra states in the second quiver then appear from the bound state of a center carrying charge $\alpha+M \gamma_{k} \max \left(0,\left\langle\alpha, \gamma_{k}\right\rangle\right)$ and other states with charges proportional to $-\gamma_{k}$. This in turn justifies the transformation law of $\Omega_{\mathrm{S}}$ given in the first equation of (1.13).

This transformation law is also consistent with the requirement that a monodromy, exemplified in figure 1, leaves invariant the physical properties of the BPS spectrum. Since the monodromy transformation is induced by successive application of two mutations, one with a node carrying charge proportional to $\gamma_{k}$ and then with a node carrying charges proportional to $-\gamma_{k}$, the transformation law (1.13) under a mutation implies that under a monodromy we have $\tilde{\Omega}_{\mathrm{S}}\left(\alpha+M\left\langle\alpha, \gamma_{k}\right\rangle \gamma_{k}\right)=\Omega_{\mathrm{S}}(\alpha)$, where we denoted by $\tilde{\Omega}_{\mathrm{S}}$ the single centered indices after the monodromy transformation. On the other hand a monodromy maps a BPS bound state with constituent charges $\alpha$ to one with charges $\tilde{\alpha}=\alpha+M\left\langle\alpha, \gamma_{k}\right\rangle \gamma_{k}$, while other physical quantities as the central charges and symplectic inner products remain invariant. Moreover, the physical equivalence of the bound states before and after the monodromy requires that the single centered indices transform as $\tilde{\Omega}_{\mathrm{S}}(\tilde{\alpha})=\Omega_{\mathrm{S}}(\alpha)$. This agrees with the monodromy transformation law of $\Omega_{\mathrm{S}}$ obtained by application of two successive mutations.

\subsection{Dependence on the choice of FI parameters}

Note that while (1.12) fixes the sign of $\zeta_{k}$, it leaves unfixed the signs and the magnitudes of the other $\zeta_{i}$ 's as long as they satisfy $\sum_{i} N_{i} \zeta_{i}=0$. Since for different choices of the FI parameters we have different $Q_{\text {Coulomb }}$ and $Q_{\text {Coulomb }}^{\prime},(1.10)$ apparently gives different consistency relations for different choices of FI parameters. We shall now outline a proof that once the mutation invariance has been tested for one choice of FI parameters, its validity for other choices of FI parameters subject to the restriction (1.12) is automatic. We shall carry out the proof in steps.

First consider a vector $\gamma \in \Gamma^{+} \backslash \Gamma^{\prime+}$ (i.e. such that $\gamma=\sum_{i} n_{i} \gamma_{i}=\sum_{i} n_{i}^{\prime} \gamma_{i}^{\prime}$ with nonnegative $n_{i}$ 's, but with some negative $n_{k}^{\prime}$ ). In this case $Q^{\prime}(\gamma)$ (and the rational invariant $\bar{Q}^{\prime}(\gamma)$ ) vanishes in all chambers and hence $Q(\gamma)$ and $\bar{Q}(\gamma)$ must also vanish in all chambers. We shall now prove that it is enough to check that $\bar{Q}(\gamma)$ vanishes in any one chamber, by induction on the rank $r=\sum n_{i} .{ }^{8}$ Suppose that we have verified the vanishing of $\bar{Q}(\gamma)$ for all $\gamma \in \Gamma^{+} \backslash \Gamma^{\prime+}$ with rank $\leq r_{0}$ for some integer $r_{0}$. Now consider a $\gamma \in \Gamma^{+} \backslash \Gamma^{\prime+}$ with rank $r=r_{0}+1$, and suppose that $\bar{Q}(\gamma)$ vanishes in some chamber $c_{+}$. If we now go across a wall of $c_{+}$then the jump in $\bar{Q}(\gamma)$ across the wall will be given by the sum of products of $\bar{Q}\left(\alpha_{i}\right)$ for appropriate charge vectors $\alpha_{i}$ satisfying $\sum_{i} \alpha_{i}=\gamma$. Now in the original quiver each of the $\alpha_{i}$ 's have rank less than $r_{0}$. Furthermore at least one of the $\alpha_{i}$ 's must be in $\gamma \in \Gamma^{+} \backslash \Gamma^{\prime+}$; to see this note that when we express $\gamma=\sum_{i} \alpha_{i}$ in the $\gamma_{i}^{\prime}$ basis the coefficient of $\gamma_{k}^{\prime}$ is negative, and hence at least one of the $\alpha_{i}^{\prime}$ 's expressed in the $\gamma_{i}^{\prime}$ basis has negative coefficient of $\gamma_{k}^{\prime}$. Thus the corresponding $\bar{Q}\left(\alpha_{i}\right)$ vanishes by assumption, causing the net jump in $\bar{Q}(\gamma)$ to vanish. Thus the vanishing of $\bar{Q}(\gamma)$ in one chamber implies its vanishing in all chambers. Similarly, if $\gamma \in \Gamma^{\prime+} \backslash \Gamma^{+}$, the same argument shows that the vanishing of $Q^{\prime}(\gamma)$ in one chamber is sufficient to ensure the vanishing in all chambers.

\footnotetext{
${ }^{8}$ Note that the rank depends on whether we are using the original or the mutated quiver. Here rank will refer to the rank in the original quiver.
} 
Now suppose that we have already established the vanishing of $Q(\gamma)$ for $\gamma \in \Gamma^{+} \backslash \Gamma^{\prime+}$ and of $Q^{\prime}(\gamma)$ for $\gamma \in \Gamma^{\prime+} \backslash \Gamma^{+}$in all the chambers subject to the restriction (1.12). We now consider a general charge vector $\gamma$. Our goal will be to show that to test the equivalence of $Q(\gamma)$ and $Q^{\prime}(\gamma)$, it is enough to verify this in one chamber for each $\gamma$. We shall carry out this proof by induction. Let us suppose that we have established the equality of $Q(\gamma)$ and $Q^{\prime}(\gamma)$ for all $\gamma$ (except for $\gamma \| \gamma_{k}$ ) of rank $\leq r_{0}$ in the $\gamma_{i}$ basis in all chambers subject to the restriction (1.12). We shall then prove that for a charge vector $\gamma$ of rank $r_{0}+1$, the equality of $Q(\gamma)$ and $Q^{\prime}(\gamma)$ in any one chamber $c_{+}$implies their equality in all chambers. For this consider a wall of marginal stability that forms a boundary of $c_{+}$. Then as we approach this wall we can find a pair of primitive charge vectors $\alpha_{1}$ and $\alpha_{2}$ such that $\gamma=M_{1} \alpha_{1}+M_{2} \alpha_{2}$ for positive integer $M_{1}$ and $M_{2}$ and furthermore the FI parameters associated with the vectors $\alpha_{1}$ and $\alpha_{2}$ change sign across the wall. Using the wall-crossing formula, the jump in $Q(\gamma)$ across the wall can be expressed as a sum of products of $\bar{Q}\left(m \alpha_{1}+n \alpha_{2}\right)$ for integer $m, n$ in appropriate chambers relevant for those quivers. Similarly the jump in $Q^{\prime}(\gamma)$ can be expressed as a sum of products of $Q^{\prime}\left(m \alpha_{1}+n \alpha_{2}\right)$ for positive integer $m, n$ in the same chambers using the same wall-crossing formula. Now since $m \alpha_{1}+n \alpha_{2}$, being a constituent of the charge vector $\gamma$, must have rank $<r_{0}$ in the original quiver, the equality of $Q\left(m \alpha_{1}+n \alpha_{2}\right)$ and $Q^{\prime}\left(m \alpha_{1}+n \alpha_{2}\right)$ in any chamber holds by assumption. This shows that the net jumps in $Q(\gamma)$ and $Q^{\prime}(\gamma)$ across the wall agree and hence $Q(\gamma)=Q^{\prime}(\gamma)$ on the other side of the wall.

There are two possible caveats in this argument. First we have to assume that none of the constituents carrying charge $m \alpha_{1}+n \alpha_{2}$ has charge proportional to $\gamma_{k}$ since the equality of $Q(\gamma)$ and $Q^{\prime}(\gamma)$ does not hold for these charge vectors. This is guaranteed as long as we do not cross the $\zeta_{k}=0$ wall, 1.e. as long as we obey the constraint (1.12). Second, we have implicitly assumed that for every possible set of constituents ${ }^{9}$ in the first quiver there is a corresponding set of constituents in the second quiver carrying the same index and vice versa. This is not true in general since there may be constituents in the first quiver whose image in the second quiver may contain one of more $\alpha_{i}$ 's with negative coefficient of $\gamma_{k}^{\prime}$ and hence is not a part of the second quiver. These are the $\alpha_{i}$ 's belonging to $\Gamma^{+} \backslash \Gamma^{\prime+}$. The reverse is also possible. However since we have assumed that the vanishing of $Q\left(\alpha_{i}\right)=0$ for all $\alpha_{i} \in \Gamma^{+} \backslash \Gamma^{\prime+}$ and the vanishing of $Q^{\prime}\left(\alpha_{i}\right)=0$ for all $\alpha_{i} \in \Gamma^{\prime+} \backslash \Gamma^{+}$has already been established, these possible non-matching contributions vanish identically and we get the equality of $Q(\gamma)$ and $Q^{\prime}(\gamma)$ in all chambers. This establishes that, for any $\gamma \in \Gamma$, the equality of $Q(\gamma)$ and $Q^{\prime}(\gamma)$ in all chambers follows from the equality in any given chamber.

We end by giving a physical motivation for the restriction on the FI parameters given in (1.12). As explained earlier, in $\mathcal{N}=2$ supersymmetric theories where quiver invariants capture the index of BPS states, the mutation $\mu_{k}^{+}$takes place on walls where the central charge $Z\left(\gamma_{k}\right)$ leaves the half-plane distinguishing BPS states from anti-BPS states, while $Z\left(-\gamma_{k}\right)$ enters the same half-plane. This clearly requires that in the complex plane the ray of $Z\left(\gamma_{k}\right)$ lies to the extreme left of the ray of any other $Z(\gamma)$ inside the BPS half-plane. Now

\footnotetext{
${ }^{9}$ Here by constituent we do not mean only single-centered constituents but also bound systems whose single-centered constituents remain at finite separation as we approach the wall. The index carried by such a constituent of charge $\alpha$ is given by $Q(\alpha)$ in appropriate chamber.
} 
the FI parameter associated with $\gamma_{k}$ for a particular quiver of total charge $\gamma$ is given by

$$
\zeta_{k}=\operatorname{Im}\left(Z\left(\gamma_{k}\right) / Z(\gamma)\right)
$$

The condition on $Z\left(\gamma_{k}\right)$ mentioned above requires that $\zeta_{k}$ is negative. However it does not specify its magnitude, nor the magnitude or signs of the $\zeta_{i}$ 's carried the other constituents, as those depend both on the phases of $Z\left(\gamma_{i}\right)$ and on their magnitudes. Thus we see from this physical consideration that if mutation is to be a symmetry, it must hold under the condition (1.12) with no further constraint on the other $\zeta_{i}$ 's.

\section{Examples of ordinary quiver mutations}

In this section we shall test mutation invariance of the Coulomb branch formula for ordinary quivers. For this we take $\Omega_{\mathrm{S}}(\gamma)$ to satisfy (1.6) and use the transformation law (1.13) of $\Omega_{\mathrm{S}}(\gamma)$ under mutation. We also use mutation invariance to compute single-centered indices for various quivers where a direct analysis of the Higgs branch is forbidding. Since ordinary mutation is known to be a symmetry of the quiver Poincaré polynomial, the analysis of this section can be interpreted as a test of the Coulomb branch formula (1.3), (1.4) and the transformation rule (1.13) for single-centered indices.

Example 1. Consider a 3-node quiver with charge vectors $\gamma_{1}, \gamma_{2}$ and $\gamma_{3}$ associated with the nodes satisfying

$$
\gamma_{12}=a, \quad \gamma_{23}=b, \quad \gamma_{31}=c, \quad \zeta_{1}<0, \quad \zeta_{2}, \zeta_{3}>0, \quad a, b, c>0 .
$$

Then mutation with respect to the node 1 generates a new quiver with basis vectors

$$
\gamma_{1}^{\prime}=-\gamma_{1}, \quad \gamma_{2}^{\prime}=\gamma_{2}, \quad \gamma_{3}^{\prime}=\gamma_{3}+c \gamma_{1},
$$

DSZ matrix

$$
\gamma_{12}^{\prime}=-a, \quad \gamma_{23}^{\prime}=b-a c, \quad \gamma_{31}^{\prime}=-c,
$$

FI parameters

$$
\zeta_{1}^{\prime}=-\zeta_{1}, \quad \zeta_{2}^{\prime}=\zeta_{2}, \quad \zeta_{3}^{\prime}=\zeta_{3}+c \zeta_{1}
$$

and dimension vector

$$
\gamma=N_{1}^{\prime} \gamma_{1}^{\prime}+N_{2}^{\prime} \gamma_{2}^{\prime}+N_{3}^{\prime} \gamma_{3}^{\prime}, \quad N_{1}^{\prime}=c N_{3}-N_{1}, \quad N_{2}^{\prime}=N_{2}, \quad N_{3}^{\prime}=N_{3}
$$

The original and mutated quiver are depicted in figure 2 .

Mutation invariance (1.10) requires

$$
Q\left(N_{1}, N_{2}, N_{3}\right)=Q^{\prime}\left(N_{1}^{\prime}, N_{2}^{\prime}, N_{3}^{\prime}\right),
$$

where the l.h.s. is the shorthand notation for $Q_{\text {Coulomb }}\left(\sum_{i=1}^{3} N_{i} \gamma_{i} ; \zeta ; y ; t\right)$ while the r.h.s. is the shorthand notation for $Q_{\text {Coulomb }}^{\prime}\left(\sum_{i=1}^{3} N_{i}^{\prime} \gamma_{i}^{\prime} ; \zeta^{\prime} ; y ; t\right)$, computed with $\gamma_{i}^{\prime}$ as the basis 



Figure 2. The original quiver (left) and the mutated quiver (right) of examples 1 and 2 . .

vectors and hence $\gamma_{i j}^{\prime}$ as the DSZ products. We shall also use $\Omega_{\mathrm{S}}\left(N_{1}, N_{2}, N_{3}\right)$ to denote $\Omega_{\mathrm{S}}\left(\sum_{i=1}^{3} N_{i} \gamma_{i}\right)$ and $\Omega_{\mathrm{S}}^{\prime}\left(N_{1}^{\prime}, N_{2}^{\prime}, N_{3}^{\prime}\right)$ to denote $\Omega_{\mathrm{S}}^{\prime}\left(\sum_{i=1}^{3} N_{i}^{\prime} \gamma_{i}^{\prime} ; t\right)$. Eq. (1.13) then gives $\Omega_{\mathrm{S}}\left(N_{1}, N_{2}, N_{3}\right)=\Omega_{\mathrm{S}}^{\prime}\left(c N_{3}-N_{1}-\max \left(0, N_{3} c-N_{2} a\right), N_{2}, N_{3}\right)=\Omega_{\mathrm{S}}^{\prime}\left(\min \left(N_{3} c, N_{2} a\right)-N_{1}, N_{2}, N_{3}\right)$.

Let us choose

$a=3, \quad b=4, \quad c=5, \quad \zeta_{1}=-5.71, \quad \zeta_{2}=2.56 N_{1} / N_{2}+.01 / N_{2}, \quad \zeta_{3}=3.15 N_{1} / N_{3}-.01 / N_{3}$.

Then we get

$$
\begin{aligned}
& \gamma_{12}^{\prime}=-3, \quad \gamma_{23}^{\prime}=-11, \quad \gamma_{31}^{\prime}=-5, \\
& \zeta_{1}^{\prime}=5.71, \quad \zeta_{2}^{\prime}=2.56 N_{1} / N_{2}+.01 / N_{2}, \quad \zeta_{3}^{\prime}=3.15 N_{1} / N_{3}-28.55-.01 / N_{3} .
\end{aligned}
$$

Some of the relations following from (3.7) are

$$
\Omega_{\mathrm{S}}(N, 1,1)=\Omega_{\mathrm{S}}^{\prime}(3-N, 1,1), \quad \Rightarrow \quad \Omega_{\mathrm{S}}(N, 1,1)=0=\Omega_{\mathrm{S}}^{\prime}(N, 1,1) \quad \text { for } \quad N \geq 3 .
$$

We shall now check the invariance of the Coulomb branch formula under mutation. Eq. (3.6) gives

$$
Q(N, 1,1)=Q^{\prime}(5-N, 1,1) \quad \text { for } \quad 0 \leq N \leq 5, \quad Q(N, 1,1)=0=Q^{\prime}(N, 1,1) \quad \text { for } \quad N \geq 6 .
$$

Now explicit evaluation gives

$$
\begin{aligned}
Q(1,1,1) & =1 / y^{4}+2 / y^{2}+3+2 y^{2}+y^{4}+\Omega_{\mathrm{S}}(1,1,1), \\
Q^{\prime}(4,1,1) & =1 / y^{4}+2 / y^{2}+3+2 y^{2}+y^{4}+\Omega_{\mathrm{S}}^{\prime}(2,1,1)-\left(y+y^{-1}\right) \Omega_{\mathrm{S}}^{\prime}(3,1,1)+\Omega_{\mathrm{S}}^{\prime}(4,1,1) .
\end{aligned}
$$

Using (3.10) we see that $Q(1,1,1)$ and $Q^{\prime}(4,1,1)$ agree. Next we compute

$$
\begin{aligned}
Q(2,1,1) & =-\left(y^{-3}+2 y^{-1}+2 y+y^{3}\right)-\left(y^{-1}+y\right) \Omega_{\mathrm{S}}(1,1,1)+\Omega_{\mathrm{S}}(2,1,1) \\
Q^{\prime}(3,1,1) & =\Omega_{\mathrm{S}}^{\prime}(1,1,1)-\left(y^{-3}+2 y^{-1}+2 y+y^{3}\right)-\left(y^{-1}+y\right) \Omega_{\mathrm{S}}^{\prime}(2,1,1)+\Omega_{\mathrm{S}}^{\prime}(3,1,1) .
\end{aligned}
$$

Again using (3.10) we see that $Q(2,1,1)$ and $Q^{\prime}(3,1,1)$ agree. Similarly we have

$$
Q(3,1,1)=1+\Omega_{\mathrm{S}}(1,1,1)+\Omega_{\mathrm{S}}(3,1,1)-\left(y+y^{-1}\right) \Omega_{\mathrm{S}}(2,1,1),
$$




$$
Q^{\prime}(2,1,1)=1+\Omega_{\mathrm{S}}^{\prime}(2,1,1)-\left(y+y^{-1}\right) \Omega_{\mathrm{S}}^{\prime}(1,1,1) .
$$

These two agree as a consequence of (3.10). We also have

$$
\begin{aligned}
Q(4,1,1) & =\Omega_{\mathrm{S}}(2,1,1)+\Omega_{\mathrm{S}}(4,1,1)-\left(y+y^{-1}\right) \Omega_{\mathrm{S}}(3,1,1) \\
Q^{\prime}(1,1,1) & =\Omega_{\mathrm{S}}^{\prime}(1,1,1)
\end{aligned}
$$

which are in agreement as a consequence of (3.10). Finally we have

$$
\begin{aligned}
Q(5,1,1) & =\Omega_{\mathrm{S}}(3,1,1)+\Omega_{\mathrm{S}}(5,1,1)-\left(y+y^{-1}\right) \Omega_{\mathrm{S}}(4,1,1) \\
Q^{\prime}(0,1,1) & =0 \\
Q(0,1,1) & =-\left(y^{-3}+y^{-1}+y+y^{3}\right) \\
Q^{\prime}(5,1,1) & =-\left(y^{-3}+y^{-1}+y+y^{3}\right)+\Omega_{\mathrm{S}}^{\prime}(3,1,1)-\left(y+y^{-1}\right) \Omega_{\mathrm{S}}^{\prime}(4,1,1)+\Omega_{\mathrm{S}}^{\prime}(5,1,1) .
\end{aligned}
$$

Again these equations are in agreement due to (3.10). We have not tested the vanishing of $Q(N, 1,1)$ and $Q^{\prime}(N, 1,1)$ for $N \geq 6$ due to the increase in the computational time, but we shall test similar relations involving other quivers later.

So far we have not used any explicit results for $\Omega_{\mathrm{S}}$ or $\Omega_{\mathrm{S}}^{\prime}$. We now note that $\Omega_{\mathrm{S}}^{\prime}(1,1,1)$ vanishes since the corresponding $\gamma_{i j}^{\prime}$ 's fail to satisfy the triangle inequality. The single-centered index $\Omega_{\mathrm{S}}(1,1,1 ; t)=9$ is easily computed from the results in $[26,28,29]$. Thus we have

$$
\Omega_{\mathrm{S}}^{\prime}(1,1,1 ; t)=\Omega_{\mathrm{S}}(2,1,1 ; t)=0, \quad \Omega_{\mathrm{S}}^{\prime}(2,1,1 ; t)=\Omega_{\mathrm{S}}(1,1,1 ; t)=9 .
$$

It will be interesting to check the prediction for $\Omega_{\mathrm{S}}^{\prime}(2,1,1)$ by direct computation. Note that in general $\Omega_{\mathrm{S}}(\gamma) \neq \Omega_{\mathrm{S}}^{\prime}(\gamma)$. For example $4 \gamma_{1}^{\prime}+\gamma_{2}^{\prime}+\gamma_{3}^{\prime}=\gamma_{1}+\gamma_{2}+\gamma_{3}$ and $\Omega_{\mathrm{S}}^{\prime}(4,1,1) \neq \Omega_{\mathrm{S}}(1,1,1)$.

Example 2. We again consider a 3-node quiver with

$$
a=2, \quad b=2, \quad c=2, \quad \zeta_{1}=-3.1, \quad \zeta_{2}=N_{1} / N_{2}+.2 / N_{2}, \quad \zeta_{3}=2.1 N_{1} / N_{3}-.2 / N_{3},
$$

and mutate with respect to the node 1 . Then we get

$$
\begin{aligned}
& \gamma_{12}^{\prime}=-2, \quad \gamma_{23}^{\prime}=-2, \quad \gamma_{31}^{\prime}=-2, \\
& \zeta_{1}^{\prime}=3.1, \quad \zeta_{2}^{\prime}=N_{1} / N_{2}+.2 / N_{2}, \quad \zeta_{3}^{\prime}=2.1 N_{1} / N_{3}-6.2-.2 / N_{3} \text {, } \\
& N_{1}^{\prime}=2 N_{3}-N_{1}, \quad N_{2}^{\prime}=N_{2}, \quad N_{3}^{\prime}=N_{3} \text {. }
\end{aligned}
$$

Eqs. (3.7) give

$$
\Omega_{\mathrm{S}}\left(N_{1}, N_{2}, N_{3}\right)=\Omega_{\mathrm{S}}^{\prime}\left(\min \left(2 N_{3}, 2 N_{2}\right)-N_{1}, N_{2}, N_{3}\right) .
$$

On the other hand since the new quiver is the same as the old one with the arrows reversed and different FI parameters, and since $\Omega_{\mathrm{S}}$ is independent of the FI parameters we have

$$
\Omega_{\mathrm{S}}^{\prime}\left(N_{1}, N_{2}, N_{3}\right)=\Omega_{\mathrm{S}}\left(N_{3}, N_{2}, N_{1}\right)
$$


Furthermore cyclic invariance of the quiver implies that $\Omega_{\mathrm{S}}\left(N_{1}, N_{2}, N_{3}\right)$ is invariant under cyclic permutations of $\left(N_{1}, N_{2}, N_{3}\right)$. Using these relations we can severely constrain the values of $\Omega_{\mathrm{S}}$. For example we have ${ }^{10}$

$$
\Omega_{\mathrm{S}}(N, 1,1)=\Omega_{\mathrm{S}}^{\prime}(2-N, 1,1)=\Omega_{\mathrm{S}}(1,1,2-N)=\Omega_{\mathrm{S}}(2-N, 1,1),
$$

and as a consequence

$$
\Omega_{\mathrm{S}}(N, 1,1)=0 \quad \text { for } \quad N \geq 2 .
$$

More generally we get

$$
\Omega_{\mathrm{S}}\left(N_{1}, N_{2}, N_{3}\right)=0 \quad \text { for } \quad N_{1} \geq \min \left(2 N_{2}, 2 N_{3}\right) .
$$

Together with cyclic symmetry this implies that a necessary condition for getting nonvanishing $\Omega_{\mathrm{S}}\left(N_{1}, N_{2}, N_{3}\right)$ is that each $N_{i}$ should be strictly less than the double of each of the other two $N_{i}$ 's. Using cyclic symmetry we can take $N_{1}$ to be the largest of $\left(N_{1}, N_{2}, N_{3}\right)$. The mutation rule (3.22) then equates $\Omega_{\mathrm{S}}\left(N_{1}, N_{2}, N_{3}\right)$ to $\Omega_{\mathrm{S}}^{\prime}\left(N_{1}^{\prime}, N_{2}, N_{3}\right)=\Omega_{\mathrm{S}}\left(N_{3}, N_{2}, N_{1}^{\prime}\right)$ with $N_{1}^{\prime} \leq N_{1}$. The equality sign holds only if $N_{1}=N_{2}=N_{3}$. Thus unless $N_{1}=N_{2}=N_{3}$ we can repeatedly use mutation and cyclic symmetry to reduce the rank of the quiver until the maximum $N_{i}$ becomes greater than or equal to twice the minimum $N_{i}$, and then $\Omega_{\mathrm{S}}$ vanishes by (3.26). Thus the only non-vanishing $\Omega_{\mathrm{S}}$ in this case are $\Omega_{\mathrm{S}}(N, N, N)$. We know from [26] that in the Abelian case, $\Omega_{\mathrm{S}}(1,1,1 ; t)=1$.

We now proceed to test the invariance of the Coulomb branch formula under mutation. From the general equation $Q\left(N_{1}, N_{2}, N_{3}\right)=Q\left(2 N_{3}-N_{1}, N_{2}, N_{3}\right)$ that follows from (3.21), we get in particular

$$
Q(N, 1,1)=Q(2-N, 1,1) \quad \Rightarrow \quad Q(N, 1,1)=0 \quad \text { for } \quad N \geq 3 .
$$

Explicit calculation gives

$$
\begin{aligned}
Q(1,1,1) & =1+\Omega_{\mathrm{S}}(1,1,1), & & Q^{\prime}(1,1,1)=1+\Omega_{\mathrm{S}}^{\prime}(1,1,1), \\
Q(2,1,1) & =\Omega_{\mathrm{S}}(2,1,1), & & Q^{\prime}(0,1,1)=0, \\
Q(0,1,1) & =-\left(y+y^{-1}\right), & & Q^{\prime}(2,1,1)=-\left(y+y^{-1}\right)+\Omega_{\mathrm{S}}^{\prime}(2,1,1), \\
Q(3,1,1) & =\Omega_{\mathrm{S}}(3,1,1), & & Q(4,1,1)=\Omega_{\mathrm{S}}(4,1,1), \\
Q^{\prime}(3,1,1) & =\Omega_{\mathrm{S}}^{\prime}(3,1,1), & Q^{\prime}(4,1,1) & =\Omega_{\mathrm{S}}^{\prime}(4,1,1) .
\end{aligned}
$$

These results are all consistent with (3.27) after we use eqs. (3.24), (3.25).

More generally, for any 3-node quiver with $a, b>0$ and $c=2$, the Abelian representation $(1,1,1)$ is mapped by a mutation on node 1 to an Abelian representation. We know from the analysis of $\Omega_{\mathrm{S}}$ for $\vec{N}=(1,1,1)$ given in [26-29], that the only non-vanishing $\Omega_{\mathrm{S}}$ arise for $a=b \geq 2$. In this case (3.7) gives

$$
\Omega_{\mathrm{S}}(N, 1,1)=\Omega_{\mathrm{S}}^{\prime}(2-N, 1,1) .
$$

\footnotetext{
${ }^{10}$ The fact that $\Omega_{\mathrm{S}}(N, 1,1)$ vanishes is consistent with the fact that in the chamber $\zeta_{2}>0, \zeta_{1} \rightarrow 0^{-}$the moduli space is a codimension $N a$ surface in $\mathbb{P}^{b-1} \times G(N, c)$, with dimension $1-N^{2}$.
} 
In particular $\Omega_{\mathrm{S}}(1,1,1)=\Omega_{\mathrm{S}}^{\prime}(1,1,1)$. On the other hand since in each of these cases the arrow multiplicities computed using (3.2) are just reversed under the mutation, the equality of $\Omega_{\mathrm{S}}(1,1,1)$ and $\Omega_{\mathrm{S}}^{\prime}(1,1,1)$ follows automatically, confirming the transformation laws of $\Omega_{\mathrm{S}}$ under mutation. Using this we can verify the equality of $Q(1,1,1)$ and $Q^{\prime}(1,1,1)$.

Example 3. Next we consider the 4-node quiver



with multiplicities of the arrows $a=5, b=5, c=2$ and $d=1$. We choose for the FI parameters

$$
\vec{\zeta}=\left(\frac{25 N_{4}+.1}{N_{1}}, \frac{17 N_{4}+.2}{N_{2}}, \frac{3 N_{4}-.3}{N_{3}},-45\right) .
$$

We now perform a mutation at node 4 . The mutated quiver is:



with

$$
\begin{aligned}
\gamma_{1}^{\prime} & =\gamma_{1}, \quad \gamma_{2}^{\prime}=\gamma_{2}, \quad \gamma_{3}^{\prime}=\gamma_{3}+2 \gamma_{4}, \quad \gamma_{4}^{\prime}=-\gamma_{4}, \\
\vec{\zeta}^{\prime} & =\left(\frac{25 N_{4}+.1}{N_{1}}, \frac{17 N_{4}+.2}{N_{2}}, \frac{3 N_{4}-.3}{N_{3}}-90,45\right), \\
N_{1}^{\prime} & =N_{1}, \quad N_{2}^{\prime}=N_{2}, \quad N_{3}^{\prime}=N_{3}, \quad N_{4}^{\prime}=c N_{3}-N_{4}=2 N_{3}-N_{4} .
\end{aligned}
$$

Note that the multiplicity $c$ is chosen such that the Abelian representation $\vec{N}=(1,1,1,1)$ is mapped to the Abelian representation $\vec{N}^{\prime}=(1,1,1,1)$. More generally eq. (1.10) implies

$$
Q\left(N_{1}, N_{2}, N_{3}, N_{4}\right)=Q^{\prime}\left(N_{1}, N_{2}, N_{3}, c N_{3}-N_{4}\right)=Q^{\prime}\left(N_{1}, N_{2}, N_{3}, 2 N_{3}-N_{4}\right) .
$$

Thus we should have

$$
\begin{aligned}
Q(1,1,1,0) & =Q^{\prime}(1,1,1,2), \quad Q(1,1,1,1)=Q^{\prime}(1,1,1,1), \quad Q(1,1,1,2)=Q^{\prime}(1,1,1,0) \\
Q(1,1,1, N) & =0=Q^{\prime}(1,1,1, N) \quad \text { for } \quad N \geq 3 .
\end{aligned}
$$

In order to test this we need to first study the transformation law of $\Omega_{\mathrm{S}}$. Eq. (1.13) gives

$$
\begin{aligned}
& \Omega_{\mathrm{S}}\left(N_{1}, N_{2}, N_{3}, N_{4}\right)=\Omega_{\mathrm{S}}^{\prime}\left(N_{1}, N_{2}, N_{3}, c N_{3}-N_{4}-\max \left(c N_{3}-d N_{1}, 0\right)\right) \\
& \quad=\Omega_{\mathrm{S}}^{\prime}\left(N_{1}, N_{2}, N_{3}, \min \left(c N_{3}, d N_{1}\right)-N_{4}\right)=\Omega_{\mathrm{S}}^{\prime}\left(N_{1}, N_{2}, N_{3}, \min \left(2 N_{3}, N_{1}\right)-N_{4}\right) .
\end{aligned}
$$


This gives in particular

$$
\begin{aligned}
\Omega_{\mathrm{S}}(1,1,1,1) & =\Omega_{\mathrm{S}}^{\prime}(1,1,1,0), \quad \Omega_{\mathrm{S}}(1,1,1,0)=\Omega_{\mathrm{S}}^{\prime}(1,1,1,1) \\
\Omega_{\mathrm{S}}(1,1,1, N) & =0=\Omega_{\mathrm{S}}^{\prime}(1,1,1, N) \quad \text { for } \quad N \geq 2 .
\end{aligned}
$$

We now proceed to verify (3.37). One finds using (1.3):

$$
\begin{aligned}
& Q(1,1,1,0)=1 / y^{8}+2 / y^{6}+3 / y^{4}+4 / y^{2}+5+4 y^{2}+3 y^{4}+2 y^{6}+y^{8} \\
& Q(1,1,1,1)=y^{-8}+3 y^{-6}+5 y^{-4}+7 y^{-2}+9+7 y^{2}+5 y^{4}+3 y^{6}+y^{8}+\Omega_{\mathrm{S}}(1,1,1,1) \\
& Q(1,1,1,2)=y^{-6}+2 y^{-4}+3 y^{-2}+4+3 y^{2}+2 y^{4}+y^{6}+\Omega_{\mathrm{S}}(1,1,1,1)+\Omega_{\mathrm{S}}(1,1,1,2) \\
& Q(1,1,1,3)=\Omega_{\mathrm{S}}(1,1,1,2)+\Omega_{\mathrm{S}}(1,1,1,3) \\
& Q(1,1,1,4)=\Omega_{\mathrm{S}}(1,1,1,3)+\Omega_{\mathrm{S}}(1,1,1,4)
\end{aligned}
$$

and

$$
\begin{aligned}
Q^{\prime}(1,1,1,2)= & 1 / y^{8}+2 / y^{6}+3 / y^{4}+4 / y^{2}+5+4 y^{2}+3 y^{4}+2 y^{6}+y^{8} \\
& +\Omega_{\mathrm{S}}^{\prime}(1,1,1,1)+\Omega_{\mathrm{S}}^{\prime}(1,1,1,2) \\
Q^{\prime}(1,1,1,1)= & y^{-8}+3 y^{-6}+5 y^{-4}+7 y^{-2}+9+7 y^{2}+5 y^{4}+3 y^{6}+y^{8} \\
& +\Omega_{\mathrm{S}}^{\prime}(1,1,1,0)+\Omega_{\mathrm{S}}^{\prime}(1,1,1,1) \\
Q^{\prime}(1,1,1,0)= & y^{-6}+2 y^{-4}+3 y^{-2}+4+3 y^{2}+2 y^{4}+y^{6}+\Omega_{\mathrm{S}}^{\prime}(1,1,1,0) .
\end{aligned}
$$

Compatibility of these expressions with (3.40), (3.37) follows directly from (3.39) and (3.43). In particular the last two equations of (3.40) are consistent with (3.37), (3.39). We can also test the vanishing of $Q^{\prime}(1,1,1, N)$ for $N \geq 3$. For $\zeta^{\prime}$ given by (3.34) with $\left(N_{1}, N_{2}, N_{3}, N_{4}\right)=(1,1,1,2-N)$, we get

$$
\begin{aligned}
& Q^{\prime}(1,1,1,3)=\Omega_{\mathrm{S}}^{\prime}(1,1,1,2)+\Omega_{\mathrm{S}}^{\prime}(1,1,1,3), \\
& Q^{\prime}(1,1,1,4)=\Omega_{\mathrm{S}}^{\prime}(1,1,1,3)+\Omega_{\mathrm{S}}^{\prime}(1,1,1,4) .
\end{aligned}
$$

These vanish using (3.39).

Note that in the above analysis we have not explicitly used the values of $\Omega_{\mathrm{S}}$ and $\Omega_{\mathrm{S}}^{\prime}$ or tested (3.39). From direct analysis of 3-node and 4-node cyclic quiver given in [26-29] we know that $\Omega_{\mathrm{S}}(1,1,1,0 ; t)=0$ (as there is no loop) and $\Omega_{\mathrm{S}}(1,1,1,1 ; t)=4$. Thus we have

$$
\Omega_{\mathrm{S}}^{\prime}(1,1,1,1 ; t)=\Omega_{\mathrm{S}}(1,1,1,0 ; t)=0, \quad \Omega_{\mathrm{S}}^{\prime}(1,1,1,0 ; t)=\Omega_{\mathrm{S}}(1,1,1,1 ; t)=4 .
$$

The value of $\Omega_{\mathrm{S}}^{\prime}(1,1,1,0 ; t)$ given in [26-29] agrees with the result given above. Vanishing of $\Omega_{\mathrm{S}}^{\prime}(1,1,1,1 ; t)$ can be seen by direct analysis of the Higgs branch moduli space of this quiver.

\section{Examples of generalized quiver mutations}

In this section we test the conjectured invariance of the Coulomb branch formula for generalized quivers where the condition (1.6) is relaxed. 
Example 1. We consider the generalized Kronecker quiver with $m \equiv \gamma_{12}>0$ arrows from node 1 to node 2 , with $\Omega_{\mathrm{S}}\left(k \gamma_{1} ; y ; t\right)$ and $\Omega_{\mathrm{S}}\left(\ell \gamma_{2} ; y ; t\right)$ given by arbitrary symmetric Laurent polynomials and $\Omega_{\mathrm{S}}(\gamma)=0$ otherwise. In the chamber $\zeta_{1}<0<\zeta_{2}$ the total index for charge $\gamma$ coincides with $\Omega_{\mathrm{S}}(\gamma)$ as there are no bound states with two or more centers. The index in the other chamber $\zeta_{1}>0>\zeta_{2}$, which we shall denote by $Q\left(N_{1}, N_{2}\right)$, can be obtained using the wall-crossing formula. We shall define, as in (1.3),

$$
\begin{aligned}
\bar{\Omega}_{\mathrm{S}}(\gamma ; y ; t) & =\sum_{m \mid \gamma} \frac{1}{m} \frac{y-y^{-1}}{y^{m}-y^{-m}} \Omega_{\mathrm{S}}\left(\gamma / m ; y^{m} ; t^{m}\right), \\
\bar{Q}_{\text {Coulomb }}(\gamma ; y ; t) & =\sum_{m \mid \gamma} \frac{1}{m} \frac{y-y^{-1}}{y^{m}-y^{-m}} Q_{\text {Coulomb }}\left(\gamma / m ; y^{m} ; t^{m}\right),
\end{aligned}
$$

and drop the arguments $y$ and $t$ from $\bar{\Omega}_{\mathrm{S}}$ to avoid cluttering. Using the shorthand notation $Q(p, q)$ for $Q_{\text {Coulomb }}\left(p \gamma_{1}+q \gamma_{2} ; \zeta ; y ; t\right)$ etc. the wall-crossing formula then takes the form

$$
\prod_{\substack{p, q \\ p / q \downarrow}} \exp \left[\bar{Q}(p, q) e_{p, q}\right]=\exp \left[\sum_{\ell} \bar{\Omega}_{\mathrm{S}}\left(\ell \gamma_{2}\right) e_{0, \ell}\right] \exp \left[\sum_{k} \bar{\Omega}_{\mathrm{S}}\left(k \gamma_{1}\right) e_{k, 0}\right],
$$

where $e_{p, q}$ are elements of an algebra satisfying the commutation relation

$$
\begin{aligned}
{\left[e_{p, q}, e_{p^{\prime}, q^{\prime}}\right] } & =\kappa\left(\gamma, \gamma^{\prime}\right) e_{p+p^{\prime}, q+q^{\prime}}, \quad \gamma \equiv p \gamma_{1}+q \gamma_{2}, \quad \gamma^{\prime} \equiv p^{\prime} \gamma_{1}+q^{\prime} \gamma_{2}, \\
\kappa\left(\gamma, \gamma^{\prime}\right) & \equiv \frac{(-y)^{\left\langle\gamma, \gamma^{\prime}\right\rangle}-(-y)^{-\left\langle\gamma, \gamma^{\prime}\right\rangle}}{y-y^{-1}} .
\end{aligned}
$$

The product over $p, q$ runs over non-negative integers $p, q$ and symbol $p / q \downarrow$ on the left hand side of (4.2) implies that the product is ordered such that the ratio $p / q$ decreases from left to right. If $p / q=p^{\prime} / q^{\prime}$ then the order is irrelevant since $e_{p, q}$ and $e_{p^{\prime}, q^{\prime}}$ will commute. Taking the $p=0$ terms on the left hand side to the right hand side and using the fact that $\bar{Q}(0, \ell)=\bar{\Omega}_{\mathrm{S}}\left(\ell \gamma_{2}\right)$, we can express $(4.2)$ as

$$
\prod_{\substack{p, q \\ p \neq 0, p / q \downarrow}} \exp \left[\bar{Q}(p, q) e_{p, q}\right]=\exp \left[\sum_{\ell} \bar{\Omega}_{\mathrm{S}}\left(\ell \gamma_{2}\right) e_{0, \ell}\right] \exp \left[\sum_{k} \bar{\Omega}_{\mathrm{S}}\left(k \gamma_{1}\right) e_{k, 0}\right] \exp \left[-\sum_{\ell} \bar{\Omega}_{\mathrm{S}}\left(\ell \gamma_{2}\right) e_{0, \ell}\right] .
$$

Under generalized mutation with respect to the node 2 , we have $\gamma_{12}^{\prime}=-\gamma_{12}$ and $\zeta_{1}^{\prime}<0<\zeta_{2}^{\prime}$. The effect of reversal of the sign of $\zeta_{i}^{\prime}$ 's will be to change the order of the products on both sides of (4.4). On the other hand the effect of changing the sign of $\gamma_{12}$ is that the corresponding generators $e_{p, q}^{\prime}$ which replace $e_{p, q}$ in (4.4) will satisfy a commutation relation similar to that of $e_{p, q}$ but with an extra minus sign on the right hand side. This means that $-e_{p, q}^{\prime}$ 's will satisfy the same commutation relations as $e_{p, q}$ 's. Thus we can write an equation similar to that of (4.4) with the order of products reversed on both sides, $\bar{Q}(p, q)$ replaced by $\bar{Q}^{\prime}(p, q)$ and $e_{p, q}$ replaced by $-e_{p, q}$ :

$$
\begin{aligned}
& \prod_{\substack{p, q \\
p \neq 0, p / q \uparrow}} \exp \left[-\bar{Q}^{\prime}(p, q) e_{p, q}\right]= \\
& \quad=\exp \left[\sum_{\ell} \bar{\Omega}_{\mathrm{S}}\left(\ell \gamma_{2}\right) e_{0, \ell}\right] \exp \left[-\sum_{k} \bar{\Omega}_{\mathrm{S}}\left(k \gamma_{1}\right) e_{k, 0}\right] \exp \left[-\sum_{\ell} \bar{\Omega}_{\mathrm{S}}\left(\ell \gamma_{2}\right) e_{0, \ell}\right] .
\end{aligned}
$$


Taking the inverse of this has the effect of reversing the order of the products and changing the signs of $e_{\gamma}$ 's in the exponent. The resulting equation is identical to that of (4.4) with $\bar{Q}(p, q)$ replaced by $\bar{Q}^{\prime}(p, q)$, showing that $\bar{Q}(p, q)=\bar{Q}^{\prime}(p, q)$ [22]. Mutation invariance however requires us to prove a different equality, namely $\bar{Q}^{\prime}(p, q)=\bar{Q}\left(p, M \gamma_{12} p-q\right)$ where

$$
M \equiv \sum_{\ell} \ell^{2} \Omega_{\mathrm{S}}\left(\ell \gamma_{2} ; y=1 ; t=1\right) .
$$

To proceed, we shall assume that as a consequence of (4.4) we have

$$
Q(p, q)=0 \quad \text { for } \quad q>M \gamma_{12} p .
$$

Later we shall prove this relation. Assuming this to be true, we define $p^{\prime}=p, \quad q^{\prime}=$ $M \gamma_{12} p-q$ (or equivalently $p=p^{\prime}, q=M \gamma_{12} p^{\prime}-q^{\prime}$ ) which are both non-negative for $p \geq 0$, $0 \leq q \leq M \gamma_{12} p$ and note that $p^{\prime} / q^{\prime}$ are ordered in increasing order if $p / q$ are ordered in the decreasing order. Then we can express (4.4) as

$$
\begin{aligned}
& \prod_{\substack{p^{\prime}, q^{\prime} \\
p^{\prime} \neq 0, p^{\prime} / q^{\prime} \uparrow}} \exp \left[\bar{Q}\left(p^{\prime}, M \gamma_{12} p^{\prime}-q^{\prime}\right) e_{p^{\prime}, M \gamma_{12} p^{\prime}-q^{\prime}}\right] \\
& \quad=\exp \left[\sum_{\ell} \bar{\Omega}_{\mathrm{S}}\left(\ell \gamma_{2}\right) e_{0, \ell}\right] \exp \left[\sum_{k} \bar{\Omega}_{\mathrm{S}}\left(k \gamma_{1}\right) e_{k, 0}\right] \exp \left[-\sum_{\ell} \bar{\Omega}_{\mathrm{S}}\left(\ell \gamma_{2}\right) e_{0, \ell}\right] .
\end{aligned}
$$

Since $p^{\prime}, q^{\prime}$ are dummy indices we can change them to $p, q$ on the left hand side. Furthermore notice that $e_{p, M \gamma_{12} p-q}$ 's and $-e_{p, q}$ 's have isomorphic algebra for different $p, q$. Thus we can replace $e_{p, M \gamma_{12} p-q}$ by $-e_{p, q}$ on both sides without changing the basic content of the equations. This gives

$$
\begin{aligned}
& \prod_{\substack{p, q \\
p \neq 0, p / q \uparrow}} \exp \left[-\bar{Q}\left(p, M \gamma_{12} p-q\right) e_{p, q}\right] \\
& =\exp \left[-\sum_{\ell} \bar{\Omega}_{\mathrm{S}}\left(\ell \gamma_{2}\right) e_{0,-\ell}\right] \exp \left[-\sum_{k} \bar{\Omega}_{\mathrm{S}}\left(k \gamma_{1}\right) e_{k, M \gamma_{12} k}\right] \exp \left[\sum_{\ell} \bar{\Omega}_{\mathrm{S}}\left(\ell \gamma_{2}\right) e_{0,-\ell}\right] .
\end{aligned}
$$

Thus the proof of mutation symmetry $\bar{Q}^{\prime}(p, q)=\bar{Q}\left(p, M \gamma_{12} p-q\right)$ reduces to proving the equality of the right hand sides of (4.5) and (4.9). This is the task we shall undertake now. For this we define

$$
U \equiv \exp \left[\sum_{\ell} \bar{\Omega}_{\mathrm{S}}\left(\ell \gamma_{2}\right) e_{0, \ell}\right], \quad V \equiv \exp \left[-\sum_{\ell} \bar{\Omega}_{\mathrm{S}}\left(\ell \gamma_{2}\right) e_{0,-\ell}\right],
$$

and express eqs. (4.5) and (4.9) as

$$
\prod_{\substack{p, q \\ p \neq 0, p / q \uparrow}} \exp \left[-\bar{Q}^{\prime}(p, q) e_{p, q}\right]=\prod_{k} \exp \left[-\bar{\Omega}_{\mathrm{S}}\left(k \gamma_{1}\right) U e_{k, 0} U^{-1}\right],
$$

and

$$
\prod_{\substack{p, q \\ p \neq 0, p / q \uparrow}} \exp \left[-\bar{Q}\left(p, M \gamma_{12} p-q\right) e_{p, q}\right]=\prod_{k} \exp \left[-\bar{\Omega}_{\mathrm{S}}\left(k \gamma_{1}\right) V e_{k, M \gamma_{12} k} V^{-1}\right] .
$$


Note that the order of terms in the product over $k$ on the right hand sides of these two equations is irrelevant since the terms for different $k$ commute. Thus the equality of the right hand side of the two expressions require us to prove that $U e_{k, 0} U^{-1}=V e_{k, M \gamma_{12} k} V^{-1}$.

Now suppose we combine all the factors on either side of (4.11) and (4.12) using the Baker-Campbell-Hausdorff formula, and consider the coefficients of $e_{1, s}$ in the exponent. On the left hand sides of (4.11) and (4.12), these are determined in terms of $\bar{Q}^{\prime}(1, q)$ and $\bar{Q}\left(1, M \gamma_{12}-q\right)$ respectively. Since we have already proved the equality of $\bar{Q}^{\prime}(1, q)$ and $\bar{Q}\left(1, M \gamma_{12}-q\right)$ with the help of semi-primitive wall-crossing formula, we see that the coefficients of $e_{1, s}$ in the exponent on the left hand sides are equal. On the other hand since $U e_{k, 0} U^{-1}$ and $V e_{k, M \gamma_{12} k} V^{-1}$ are linear combinations of $e_{k, q}$, on the right hand sides the coefficient of $e_{1, s}$ in the exponents are given by the terms proportional to $U e_{1,0} U^{-1}$ and $V e_{1, M \gamma_{12}} V^{-1}$, respectively. Thus the equality of the coefficients of $e_{1, s}$ in the exponent of the two left hand sides imply that

$$
U e_{1,0} U^{-1}=V e_{1, M \gamma_{12}} V^{-1} .
$$

Now note that if we had considered a Kronecker quiver with nodes carrying charges $k \gamma_{1}$ for fixed $k$ and and $\ell \gamma_{2}$ for different $\ell>0$, the semi-primitive wall-crossing formula would have given the equality of this with a quiver whose nodes carry charges $k \gamma_{1}+M \gamma_{12} k \gamma_{2}$ and $-\ell \gamma_{2}$ for dimension vector $(1, N)$. On the other hand such a quiver is equivalent to the one we are considering with $\Omega_{\mathrm{S}}\left(r \gamma_{1}\right)=0$ for $r \neq k$, and we can use (4.11), (4.12) for such a quiver. In this case $\bar{Q}(p, q)$ and $\bar{Q}^{\prime}\left(p, M \gamma_{12} p-q\right)$ would vanish for $1 \leq p \leq k-1$ and for $p=k$ they would be equal due to the generalized mutation invariance of the rank $(1, N)$ quiver. On the right hand sides of the corresponding eqs. (4.11) and (4.12) the $e_{k, q}$ in the exponent come from the $U e_{k, 0} U^{-1}$ and $V e_{k, M \gamma_{12} k} V^{-1}$ terms, with $U$ and $V$ given by the same expressions (4.10) as the original quivers. Thus we conclude that

$$
U e_{k, 0} U^{-1}=V e_{k, M \gamma_{12} k} V^{-1} .
$$

Since this is valid for every $k$, we see that the right hand sides of (4.11) and (4.12) are equal for the original quiver. This in turn proves the equality of the left hand sides and hence the desired relation

$$
\bar{Q}\left(p, M \gamma_{12} p-q\right)=\bar{Q}^{\prime}(p, q) .
$$

Finally, we prove (4.7) as follows. From the analysis of the rank $(1, N)$ case we know that $\bar{Q}^{\prime}(1, q)$ vanishes for $q>M \gamma_{12}$. With the help of (4.11) we can translate this to a statement that $U e_{1,0} U^{-1}$ is a linear combination of $e_{1, q}$ for $0 \leq q \leq M \gamma_{12}$. Generalizing this to the quiver whose nodes carry charges $k \gamma_{1}$ and $\gamma_{2}$ we can conclude that $U e_{k, 0} U^{-1}$ is a linear combination of $e_{k, q}$ for $0 \leq q \leq M \gamma_{12} k$. Eq. (4.11) then shows that $\bar{Q}^{\prime}(p, q)$ vanishes for $q>M \gamma_{12} p$. Equality of $Q(p, q)$ and $Q^{\prime}(p, q)$, discussed below (4.5) independent of the validity of generalized mutation symmetry, then leads to (4.7).

We shall now test this for some specific choices of single-centered indices, namely

$$
\Omega_{\mathrm{S}}\left(\gamma_{1}\right)=p_{1}, \quad \Omega_{\mathrm{S}}\left(\gamma_{2}\right)=q_{1}, \quad \Omega_{\mathrm{S}}\left(2 \gamma_{2}\right)=q_{2}, \quad p_{1}, q_{1}, q_{2} \geq 0,
$$




\begin{tabular}{|r|r|r|r|}
\hline$m$ & $p_{1}, q_{1}, q_{2}$ & $\mathcal{F}(1, q)$ & $\mathcal{F}(2, q)$ \\
\hline 1 & $1,1,0$ & $1+q$ & 0 \\
1 & $1,2,0$ & $(1+q)^{2}$ & 0 \\
1 & $1,3,0$ & $(1+q)^{3}$ & $q^{3}$ \\
1 & $2,1,0$ & $2(1+q)$ & $q$ \\
1 & $2,2,0$ & $2(1+q)^{2}$ & $\left.q q^{2}\right)$ \\
1 & $2,3,0$ & $2(1+q)^{3}$ & $2 q(1-q)$ \\
1 & $3,1,0$ & $3(1+q)$ & $\left(3-6 q+14 q^{2}-6 q^{3}+3 q^{4}\right)$ \\
1 & $3,2,0$ & $3(1+q)^{2}$ & $3 q$ \\
1 & $3,3,0$ & $3(1+q)^{3}$ & $6 q\left(1-q+q^{2}\right)$ \\
\hline 2 & $1,1,0$ & $(1-q)^{2}$ & $3 q\left(3-6 q+13 q^{2}-6 q^{3}+3 q^{4}\right)$ \\
2 & $1,2,0$ & $(1-q)^{4}$ & $q\left(1+q^{2}\right)$ \\
2 & $2,1,0$ & $2(1-q)^{2}$ & $q\left(2-4 q+22 q^{2}-20 q^{3}+22 q^{4}-4 q^{5}+2 q^{6}\right)$ \\
2 & $2,2,0$ & $2(1-q)^{4}$ & $2 q\left(3-2 q+3 q^{2}\right)$ \\
\hline 3 & $1,1,0$ & $(1+q)^{3}$ & $4 q\left(3-8 q+29 q^{2}-28 q^{3}+29 q^{4}-8 q^{5}+3 q^{6}\right)$ \\
3 & $1,2,0$ & $(1+q)^{6}$ & $2 q\left(3-15 q+85 q^{2}-165 q^{3}+351 q^{4}-337 q^{5}+351 q^{6}+\cdots+3 q^{10}\right)$ \\
\hline
\end{tabular}

Table 1. Generating functions of $Q_{\text {Coulomb }}\left(\gamma_{1}+N \gamma_{2}\right)$ and $Q_{\text {Coulomb }}\left(2 \gamma_{1}+N \gamma_{2}\right)$ for the generalized Kronecker quiver with $\Omega_{\mathrm{S}}\left(\gamma_{1}\right)=p_{1}, \Omega_{\mathrm{S}}\left(\gamma_{2}\right)=q_{1}, \Omega_{\mathrm{S}}\left(2 \gamma_{2}\right)=q_{2}$. The symmetry under $q \rightarrow 1 / q$ shows mutation invariance in these cases.

with all other single-centered indices vanishing. Generalized mutation invariance with respect to the node 2 requires that the generating function

$$
\mathcal{F}\left(N_{1}, q ; y ; t\right)=\sum_{N_{2} \geq 0} Q_{\text {Coulomb }}\left(N_{1} \gamma_{1}+N_{2} \gamma_{2} ; \zeta ; y ; t\right) q^{N_{2}}
$$

satisfies the functional equation

$$
q^{m N_{1} M} \mathcal{F}\left(N_{1}, 1 / q ; y ; t\right)=\mathcal{F}\left(N_{1}, q ; y ; t\right) .
$$

where $M \equiv q_{1}+4 q_{2}>0$. This equation holds for $N_{1}=1$ by assumption. Using the generalized semi-primitive formulae established in [22], we can test this property for $N_{1}=2$ or $N_{1}=3$. For simplicity we restrict to $N_{2}=2,1 \leq m \leq 3$ and set $y=t=1$. We have computed $\mathcal{F}(2, q)$ for the values of $\left(m, p_{1}, p_{2}, q_{1}, q_{2}\right)$ displayed in table 1 , and found that (4.18) was indeed obeyed.

In this case, we can also test whether the conditions (1.11) can be relaxed. Let us set $p_{2}=q_{2}=0, m=1$ for simplicity, and try $q_{1}=-1$. The semi-primitive partition function

$$
\mathcal{F}(1, q)=\frac{p_{1}}{1+q}
$$

is multiplied by $q$ under $q \rightarrow 1 / q$ but its rank 2 counterpart, computed using the formulae in [22], is not multiplied by $q^{2}$ under $q \rightarrow 1 / q$ :

$$
\mathcal{F}(2, q)=\frac{p_{1} q\left(1-p_{1}-\left(p_{1}+1\right) q^{2}\right)}{2(1-q)^{2}\left(1-q^{4}\right)} .
$$


This illustrates the importance of the assumption that the mutating node must carry positive $\Omega_{\mathrm{S}}$.

Example 2. We consider a three node quiver of rank $\left(N_{1}, N_{2}, N_{3}\right)$ with $\gamma_{12}=\gamma_{32}=a=1$ and $\gamma_{31}=c=2$, and take the invariants $\Omega_{\mathrm{S}}\left(\ell \gamma_{1}\right)$ and $\Omega_{\mathrm{S}}\left(\ell \gamma_{3}\right)$ to be generic functions of $\ell$, $y$ and $t$ and $\Omega_{\mathrm{S}}\left(\ell \gamma_{2} ; y ; t\right)$ for different integers $\ell$ to be specific functions of $y$ and $t$ to be described below. All other $\Omega_{\mathrm{S}}(\gamma ; y ; t)$ 's will be taken to vanish. For the FI parameters, we take

$$
\zeta_{1}=\left(3 N_{2}+.1\right) / N_{1}, \quad \zeta_{2}=-8, \quad \zeta_{3}=\left(5 N_{2}-.1\right) / N_{3} .
$$

Under mutation with respect to the node 2, we get

$$
\begin{aligned}
& \gamma_{1}^{\prime}=\gamma_{1}+M \gamma_{2}, \quad \gamma_{2}^{\prime}=-\gamma_{2}, \quad \gamma_{3}^{\prime}=\gamma_{3}+M \gamma_{2}, \\
& \gamma_{12}^{\prime}=-a, \quad \gamma_{23}^{\prime}=a, \quad \gamma_{31}=c
\end{aligned}
$$

where

$$
M=\sum_{\ell \geq 1} \ell^{2} \Omega_{\mathrm{S}}\left(\ell \gamma_{2} ; y=1 ; t=1\right) .
$$

Then

$$
N_{1} \gamma_{1}+N_{2} \gamma_{2}+N_{3} \gamma_{3}=N_{1} \gamma_{1}^{\prime}+\left(M N_{1}+M N_{3}-N_{2}\right) \gamma_{2}^{\prime}+N_{3} \gamma_{3}^{\prime} .
$$

The $\Omega_{\mathrm{S}}^{\prime}$ 's for the mutated quiver are given by

$$
\Omega_{\mathrm{S}}^{\prime}\left(\ell \gamma_{1}^{\prime} ; y ; t\right)=\Omega_{\mathrm{S}}\left(\ell \gamma_{1} ; y ; t\right), \quad \Omega_{\mathrm{S}}^{\prime}\left(\ell \gamma_{3}^{\prime} ; y ; t\right)=\Omega_{\mathrm{S}}\left(\ell \gamma_{3} ; y ; t\right), \quad \Omega_{\mathrm{S}}^{\prime}\left(\ell \gamma_{2}^{\prime} ; y ; t\right)=\Omega_{\mathrm{S}}\left(\ell \gamma_{2} ; y ; t\right)
$$

Finally the FI parameters of the mutated quiver are

$$
\zeta_{1}^{\prime}=\left(3 N_{2}+.1\right) / N_{1}-8 M, \quad \zeta_{3}^{\prime}=\left(5 N_{2}-.1\right) / N_{3}-8 M, \quad \zeta_{2}^{\prime}=8 .
$$

As before we denote $Q_{\text {Coulomb }}\left(N_{1} \gamma_{1}+N_{2} \gamma_{2}+N_{3} \gamma_{3} ; \zeta ; y ; t\right)$ by $Q\left(N_{1}, N_{2}, N_{3}\right)$ and similarly for the mutated quiver. Also $\Omega_{\mathrm{S}}(\gamma)$ without any other $\operatorname{argument}$ will denote $\Omega_{\mathrm{S}}(\gamma ; y ; t)$. The expected relationship between $Q$ and $Q^{\prime}$ then takes the form:

$$
Q\left(N_{1}, N_{2}, N_{3}\right)=Q^{\prime}\left(N_{1}, M N_{1}+M N_{3}-N_{2}, N_{3}\right) .
$$

We shall now consider several choices for the single-centered indices $\Omega_{\mathrm{S}}\left(\ell \gamma_{2} ; y ; t\right)$.

(a): $\Omega_{\mathrm{S}}\left(\gamma_{2} ; y ; t\right)=2, \quad \Omega_{\mathrm{S}}\left(\ell \gamma_{2} ; y ; t\right)=0$ for $\ell>1$. In this case $M=2$, and the relation (4.28) takes the form

$$
Q\left(N_{1}, N_{2}, N_{3}\right)=Q^{\prime}\left(N_{1}, 2 N_{1}+2 N_{3}-N_{2}, N_{3}\right) .
$$

Explicit calculation gives

$$
\begin{aligned}
Q(1,2,1) & =-\left(y^{-1}+y\right)\left(y^{-2}+4+y^{2}\right) \Omega_{\mathrm{S}}\left(\gamma_{1}\right) \Omega_{\mathrm{S}}\left(\gamma_{3}\right), \\
Q^{\prime}(1,2,1) & =-\left(y^{-1}+y\right)\left(y^{-2}+4+y^{2}\right) \Omega_{\mathrm{S}}^{\prime}\left(\gamma_{1}^{\prime}\right) \Omega_{\mathrm{S}}^{\prime}\left(\gamma_{3}^{\prime}\right), \\
Q(1,3,1) & =2\left(y^{-2}+1+y^{2}\right) \Omega_{\mathrm{S}}\left(\gamma_{1}\right) \Omega_{\mathrm{S}}\left(\gamma_{3}\right), \quad Q^{\prime}(1,1,1)=2\left(y^{-2}+1+y^{2}\right) \Omega_{\mathrm{S}}^{\prime}\left(\gamma_{1}^{\prime}\right) \Omega_{\mathrm{S}}^{\prime}\left(\gamma_{3}^{\prime}\right),
\end{aligned}
$$




$$
\begin{aligned}
Q(1,4,1)= & -\left(y^{-1}+y\right) \Omega_{\mathrm{S}}\left(\gamma_{1}\right) \Omega_{\mathrm{S}}\left(\gamma_{3}\right), \quad Q^{\prime}(1,0,1)=-\left(y^{-1}+y\right) \Omega_{\mathrm{S}}^{\prime}\left(\gamma_{1}^{\prime}\right) \Omega_{\mathrm{S}}^{\prime}\left(\gamma_{3}^{\prime}\right), \\
Q(1,3,2)= & \left(y^{-6}+y^{-4}+y^{-2}+1+y^{2}+y^{4}+y^{6}\right) \\
& \times\left\{\Omega_{\mathrm{S}}\left(\gamma_{3} ; y ; t\right)^{2}-\Omega_{\mathrm{S}}\left(\gamma_{3} ; y^{2} ; t^{2}\right)-2\left(y^{-1}+y\right) \Omega_{\mathrm{S}}\left(2 \gamma_{3} ; y ; t\right)\right\} \Omega_{\mathrm{S}}\left(\gamma_{1} ; y ; t\right) \\
Q^{\prime}(1,3,2)= & \left(y^{-6}+y^{-4}+y^{-2}+1+y^{2}+y^{4}+y^{6}\right) \\
& \times\left\{\Omega_{\mathrm{S}}^{\prime}\left(\gamma_{3}^{\prime} ; y ; t\right)^{2}-\Omega_{\mathrm{S}}^{\prime}\left(\gamma_{3}^{\prime} ; y^{2} ; t^{2}\right)-2\left(y^{-1}+y\right) \Omega_{\mathrm{S}}^{\prime}\left(2 \gamma_{3}^{\prime} ; y ; t\right)\right\} \Omega_{\mathrm{S}}^{\prime}\left(\gamma_{1}^{\prime} ; y ; t\right) .
\end{aligned}
$$

These results are in agreement with the generalized mutation hypothesis (4.29).

(b): $\Omega_{\mathrm{S}}\left(\gamma_{2} ; y ; t\right)=3, \quad \Omega_{\mathrm{S}}\left(\ell \gamma_{2} ; y ; t\right)=0$ for $\ell>1$. In this case $M=3$, and the relation (4.28) takes the form

$$
Q\left(N_{1}, N_{2}, N_{3}\right)=Q^{\prime}\left(N_{1}, 3 N_{1}+3 N_{3}-N_{2}, N_{3}\right)
$$

Explicit calculation gives

$$
\begin{aligned}
Q(1,2,1) & =-3\left(y^{-3}+4 y^{-1}+4 y+y^{3}\right) \Omega_{\mathrm{S}}\left(\gamma_{1}\right) \Omega_{\mathrm{S}}\left(\gamma_{3}\right) \\
Q^{\prime}(1,4,1) & =-3\left(y^{-3}+4 y^{-1}+4 y+y^{3}\right) \Omega_{\mathrm{S}}^{\prime}\left(\gamma_{1}^{\prime}\right) \Omega_{\mathrm{S}}^{\prime}\left(\gamma_{3}^{\prime}\right), \\
Q(1,3,1) & =\left(y^{-4}+10 y^{-2}+10+10 y^{2}+y^{4}\right) \Omega_{\mathrm{S}}\left(\gamma_{1}\right) \Omega_{\mathrm{S}}\left(\gamma_{3}\right), \\
Q^{\prime}(1,3,1) & =\left(y^{-4}+10 y^{-2}+10+10 y^{2}+y^{4}\right) \Omega_{\mathrm{S}}^{\prime}\left(\gamma_{1}^{\prime}\right) \Omega_{\mathrm{S}}^{\prime}\left(\gamma_{3}^{\prime}\right), \\
Q(1,4,1) & =-3\left(y^{-3}+4 y^{-1}+4 y+y^{3}\right) \Omega_{\mathrm{S}}\left(\gamma_{1}\right) \Omega_{\mathrm{S}}\left(\gamma_{3}\right), \\
Q^{\prime}(1,2,1) & =-3\left(y^{-3}+4 y^{-1}+4 y+y^{3}\right) \Omega_{\mathrm{S}}^{\prime}\left(\gamma_{1}^{\prime}\right) \Omega_{\mathrm{S}}^{\prime}\left(\gamma_{3}^{\prime}\right),
\end{aligned}
$$

in agreement with the generalized mutation hypothesis (4.31).

(c): $\Omega_{\mathrm{S}}\left(\gamma_{2} ; y ; t\right)=y^{2}+1+y^{-2}, \quad \Omega_{\mathrm{S}}\left(\ell \gamma_{2} ; y ; t\right)=0$ for $\ell>1$. In this case $M=3$, and the relation (4.28) takes the form

$$
Q\left(N_{1}, N_{2}, N_{3}\right)=Q^{\prime}\left(N_{1}, 3 N_{1}+3 N_{3}-N_{2}, N_{3}\right)
$$

Explicit calculation gives

$$
\begin{aligned}
Q(1,2,1) & =-\left(2 y^{-5}+5 y^{-3}+8 y^{-1}+8 y+5 y^{3}+2 y^{5}\right) \Omega_{\mathrm{S}}\left(\gamma_{1}\right) \Omega_{\mathrm{S}}\left(\gamma_{3}\right), \\
Q^{\prime}(1,4,1) & =-\left(2 y^{-5}+5 y^{-3}+8 y^{-1}+8 y+5 y^{3}+2 y^{5}\right) \Omega_{\mathrm{S}}^{\prime}\left(\gamma_{1}^{\prime}\right) \Omega_{\mathrm{S}}^{\prime}\left(\gamma_{3}^{\prime}\right), \\
Q(1,3,1) & =\left(y+y^{-1}\right)^{4}\left(y^{2}+y^{-2}\right) \Omega_{\mathrm{S}}\left(\gamma_{1}\right) \Omega_{\mathrm{S}}\left(\gamma_{3}\right), \\
Q^{\prime}(1,3,1) & =\left(y+y^{-1}\right)^{4}\left(y^{2}+y^{-2}\right) \Omega_{\mathrm{S}}^{\prime}\left(\gamma_{1}^{\prime}\right) \Omega_{\mathrm{S}}^{\prime}\left(\gamma_{3}^{\prime}\right), \\
Q(1,4,1) & =-\left(2 y^{-5}+5 y^{-3}+8 y^{-1}+8 y+5 y^{3}+2 y^{5}\right) \Omega_{\mathrm{S}}\left(\gamma_{1}\right) \Omega_{\mathrm{S}}\left(\gamma_{3}\right), \\
Q^{\prime}(1,2,1) & =-\left(2 y^{-5}+5 y^{-3}+8 y^{-1}+8 y+5 y^{3}+2 y^{5}\right) \Omega_{\mathrm{S}}^{\prime}\left(\gamma_{1}^{\prime}\right) \Omega_{\mathrm{S}}^{\prime}\left(\gamma_{3}^{\prime}\right),
\end{aligned}
$$

in agreement with the generalized mutation hypothesis (4.33). 
(d): $\Omega_{\mathrm{S}}\left(\gamma_{2} ; y ; t\right)=4, \quad \Omega_{\mathrm{S}}\left(\ell \gamma_{2} ; y ; t\right)=0$ for $\ell>1$. In this case $M=4$, and the relation (4.28) takes the form

$$
Q\left(N_{1}, N_{2}, N_{3}\right)=Q^{\prime}\left(N_{1}, 4 N_{1}+4 N_{3}-N_{2}, N_{3}\right)
$$

Explicit calculation gives

$$
\begin{aligned}
Q(1,4,1) & =-\left(y^{-5}+17 y^{-3}+53 y^{-1}+53 y+17 y^{3}+y^{5}\right) \Omega_{\mathrm{S}}\left(\gamma_{1}\right) \Omega_{\mathrm{S}}\left(\gamma_{3}\right), \\
Q^{\prime}(1,4,1) & =-\left(y^{-5}+17 y^{-3}+53 y^{-1}+53 y+17 y^{3}+y^{5}\right) \Omega_{\mathrm{S}}^{\prime}\left(\gamma_{1}^{\prime}\right) \Omega_{\mathrm{S}}^{\prime}\left(\gamma_{3}^{\prime}\right) .
\end{aligned}
$$

These results are in agreement with the generalized mutation hypothesis (4.35).

(e): $\Omega_{\mathrm{S}}\left(\gamma_{2} ; y ; t\right)=t+1 / t, \quad \Omega_{\mathrm{S}}\left(\ell \gamma_{2} ; y ; t\right)=0$ for $\ell>1$. In this case $M=2$, and the relation (4.28) takes the form

$$
Q\left(N_{1}, N_{2}, N_{3}\right)=Q^{\prime}\left(N_{1}, 2 N_{1}+2 N_{3}-N_{2}, N_{3}\right)
$$

Explicit calculation gives

$$
\begin{aligned}
Q(1,2,1)= & -\left(y^{-1}+y\right)\left(t^{-2}+t^{2}+y^{-2}+2+y^{2}\right) \Omega_{\mathrm{S}}\left(\gamma_{1}\right) \Omega_{\mathrm{S}}\left(\gamma_{3}\right), \\
Q^{\prime}(1,2,1)= & -\left(y^{-1}+y\right)\left(t^{-2}+t^{2}+y^{-2}+2+y^{2}\right) \Omega_{\mathrm{S}}^{\prime}\left(\gamma_{1}^{\prime}\right) \Omega_{\mathrm{S}}^{\prime}\left(\gamma_{3}^{\prime}\right), \\
Q(1,3,1)= & \left(t^{-1}+t\right)\left(y^{-2}+1+y^{2}\right) \Omega_{\mathrm{S}}\left(\gamma_{1}\right) \Omega_{\mathrm{S}}\left(\gamma_{3}\right), \\
Q^{\prime}(1,1,1)= & \left(t^{-1}+t\right)\left(y^{-2}+1+y^{2}\right) \Omega_{\mathrm{S}}^{\prime}\left(\gamma_{1}^{\prime}\right) \Omega_{\mathrm{S}}^{\prime}\left(\gamma_{3}^{\prime}\right), \\
Q(1,4,1)= & -\left(y^{-1}+y\right) \Omega_{\mathrm{S}}\left(\gamma_{1}\right) \Omega_{\mathrm{S}}\left(\gamma_{3}\right), \quad Q^{\prime}(1,0,1)=-\left(y^{-1}+y\right) \Omega_{\mathrm{S}}^{\prime}\left(\gamma_{1}^{\prime}\right) \Omega_{\mathrm{S}}^{\prime}\left(\gamma_{3}^{\prime}\right), \\
Q(1,3,2)= & \frac{1}{2}\left(t+t^{-1}\right)\left(y^{-6}+y^{-4}+y^{-2}+1+y^{2}+y^{4}+y^{6}\right) \\
& \times\left\{\Omega_{\mathrm{S}}\left(\gamma_{3} ; y ; t\right)^{2}-\Omega_{\mathrm{S}}\left(\gamma_{3} ; y^{2} ; t^{2}\right)-2\left(y^{-1}+y\right) \Omega_{\mathrm{S}}\left(2 \gamma_{3} ; y ; t\right)\right\} \Omega_{\mathrm{S}}\left(\gamma_{1} ; y ; t\right) \\
Q^{\prime}(1,3,2)= & \frac{1}{2}\left(t+t^{-1}\right)\left(y^{-6}+y^{-4}+y^{-2}+1+y^{2}+y^{4}+y^{6}\right) \\
& \times\left\{\Omega_{\mathrm{S}}^{\prime}\left(\gamma_{3}^{\prime} ; y ; t\right)^{2}-\Omega_{\mathrm{S}}^{\prime}\left(\gamma_{3}^{\prime} ; y^{2} ; t^{2}\right)-2\left(y^{-1}+y\right) \Omega_{\mathrm{S}}^{\prime}\left(2 \gamma_{3}^{\prime} ; y ; t\right)\right\} \Omega_{\mathrm{S}}^{\prime}\left(\gamma_{1}^{\prime} ; y ; t\right) .(4.38
\end{aligned}
$$

These results are in agreement with the generalized mutation hypothesis (4.37).

(f): $\quad \Omega_{\mathrm{S}}\left(\gamma_{2} ; y ; t\right)=0, \quad \Omega_{\mathrm{S}}\left(2 \gamma_{2} ; y ; t\right)=1, \quad \Omega_{\mathrm{S}}\left(\ell \gamma_{2} ; y ; t\right)=0$ for $\ell>2$. In this case $M=4$, and the relation (4.28) takes the form

$$
Q\left(N_{1}, N_{2}, N_{3}\right)=Q^{\prime}\left(N_{1}, 4 N_{1}+4 N_{3}-N_{2}, N_{3}\right) .
$$

Explicit calculation gives

$$
\begin{aligned}
Q(1,4,1) & =-\left(y^{-5}+2 y^{-3}+4 y^{-1}+4 y+2 y^{3}+y^{5}\right) \Omega_{\mathrm{S}}\left(\gamma_{1}\right) \Omega_{\mathrm{S}}\left(\gamma_{3}\right), \\
Q^{\prime}(1,4,1) & =-\left(y^{-5}+2 y^{-3}+4 y^{-1}+4 y+2 y^{3}+y^{5}\right) \Omega_{\mathrm{S}}^{\prime}\left(\gamma_{1}^{\prime}\right) \Omega_{\mathrm{S}}^{\prime}\left(\gamma_{3}^{\prime}\right) .
\end{aligned}
$$

These results are in agreement with the generalized mutation hypothesis (4.39). 
(g): we end this series of examples with a choice of $\Omega_{\mathrm{S}}$ which violates condition i) on page 6 , but which preserves the mutation symmetry at the level of numerical DTinvariants. We mentioned earlier this possibility in section 1.2 . We take $\Omega_{\mathrm{S}}\left(\gamma_{2} ; y ; t\right)=-1$ and $\Omega_{\mathrm{S}}\left(2 \gamma_{2} ; y ; t\right)=1$. We may expect the generalized mutation to be a symmetry for $y=t=1$ since the generating functions $\mathcal{F}(\vec{N} ; q ; \zeta ; q ; 1 ; 1)$ are symmetric polynomials in $q$. In particular for this choice we have $M=3$ and hence $Q\left(N_{1}, N_{2}, N_{3}\right)$ would have to be equal to $Q^{\prime}\left(N_{1}, 3 N_{1}+3 N_{3}-N_{2}, N_{3}\right)$. We find that while this does not hold for general $y$, it does hold for $y=t=1$. For example we have $Q(1,4,1)=Q^{\prime}(1,2,1)=2$ at $y=1$.

Example 3. Now we consider a three node quiver with loop by choosing $\gamma_{12}=2$, $\gamma_{23}=1$, and $\gamma_{31}=5$. We choose $\Omega_{\mathrm{S}}\left(\gamma_{2} ; y ; t\right)=2, \Omega_{\mathrm{S}}\left(\ell \gamma_{2} ; y ; t\right)=0$ for $\ell>1$, and leave $\Omega_{\mathrm{S}}\left(N_{1} \gamma_{1}+N_{2} \gamma_{2}+N_{3} \gamma_{3} ; y ; t\right)$ arbitrary except for the constraints imposed due to the restrictions mentioned at the end of section 1 . This in particular will require $\Omega_{\mathrm{S}}$ to vanish when either $N_{1}$ or $N_{3}$ vanishes with other $N_{i}$ 's being given by positive integers. The choice of FI parameters remain the same as in (4.21):

$$
\zeta_{1}=\left(3 N_{2}+.1\right) / N_{1}, \quad \zeta_{2}=-8, \quad \zeta_{3}=\left(5 N_{2}-.1\right) / N_{3} .
$$

Under mutation with respect to the node 2 , we get

$$
\begin{aligned}
& \gamma_{1}^{\prime}=\gamma_{1}+4 \gamma_{2}, \quad \gamma_{2}^{\prime}=-\gamma_{2}, \quad \gamma_{3}^{\prime}=\gamma_{3}, \\
& N_{1} \gamma_{1}+N_{2} \gamma_{2}+N_{3} \gamma_{3}=N_{1} \gamma_{1}^{\prime}+\left(4 N_{1}-N_{2}\right) \gamma_{2}^{\prime}+N_{3} \gamma_{3}^{\prime} .
\end{aligned}
$$

The $\Omega_{\mathrm{S}}^{\prime}$ 's for the mutated quiver for charge vectors proportional to the basis vectors continue to be given by (4.26). For general charge vectors we get from (1.13)

$$
\Omega_{\mathrm{S}}\left(N_{1} \gamma_{1}+N_{2} \gamma_{2}+N_{3} \gamma_{3} ; y ; t\right)=\left\{\begin{array}{lll}
\Omega_{\mathrm{S}}^{\prime}\left(N_{1} \gamma_{1}^{\prime}+\left(2 N_{3}-N_{2}\right) \gamma_{2}^{\prime}+N_{3} \gamma_{3}^{\prime} ; y ; t\right) & \text { for } \quad 2 N_{1} \geq N_{3} \\
\Omega_{\mathrm{S}}^{\prime}\left(N_{1} \gamma_{1}^{\prime}+\left(4 N_{1}-N_{2}\right) \gamma_{2}^{\prime}+N_{3} \gamma_{3}^{\prime} ; y ; t\right) & \text { for } \quad 2 N_{1}<N_{3}
\end{array} .\right.
$$

Finally the FI parameters of the mutated quiver are

$$
\zeta_{1}^{\prime}=\left(3 N_{2}+.1\right) / N_{1}-32, \quad \zeta_{3}^{\prime}=\left(5 N_{2}-.1\right) / N_{3}, \quad \zeta_{2}^{\prime}=8 .
$$

The mutated quiver has

$$
\gamma_{12}^{\prime}=-2, \quad \gamma_{13}^{\prime}=-1, \quad \gamma_{23}^{\prime}=-1 .
$$

and the expected relation is

$$
Q\left(N_{1}, N_{2}, N_{3}\right)=Q^{\prime}\left(N_{1}, 4 N_{1}-N_{2}, N_{3}\right) .
$$

Explicit calculation gives

$$
\begin{aligned}
Q(1,2,1)= & \left(y^{-4}+5 y^{-2}+6+5 y^{2}+y^{4}\right) \Omega_{\mathrm{S}}\left(\gamma_{1} ; y ; t\right) \Omega_{\mathrm{S}}\left(\gamma_{3} ; y ; t\right) \\
& +\Omega_{\mathrm{S}}\left(\gamma_{1}+\gamma_{3} ; y ; t\right)+2 \Omega_{\mathrm{S}}\left(\gamma_{1}+\gamma_{2}+\gamma_{3} ; y ; t\right)+\Omega_{\mathrm{S}}\left(\gamma_{1}+2 \gamma_{2}+\gamma_{3} ; y ; t\right) \\
Q^{\prime}(1,2,1)= & \left(y^{-4}+5 y^{-2}+6+5 y^{2}+y^{4}\right) \Omega_{\mathrm{S}}^{\prime}\left(\gamma_{1}^{\prime} ; y ; t\right) \Omega_{\mathrm{S}}^{\prime}\left(\gamma_{3}^{\prime} ; y ; t\right) \\
& +\Omega_{\mathrm{S}}^{\prime}\left(\gamma_{1}^{\prime}+\gamma_{3}^{\prime} ; y ; t\right)+2 \Omega_{\mathrm{S}}^{\prime}\left(\gamma_{1}^{\prime}+\gamma_{2}^{\prime}+\gamma_{3}^{\prime} ; y ; t\right)+\Omega_{\mathrm{S}}^{\prime}\left(\gamma_{1}^{\prime}+2 \gamma_{2}^{\prime}+\gamma_{3}^{\prime} ; y ; t\right)
\end{aligned}
$$




$$
\begin{aligned}
& Q(1,3,1)=2\left(y^{-1}+y\right)^{2} \Omega_{\mathrm{S}}\left(\gamma_{1} ; y ; t\right) \Omega_{\mathrm{S}}\left(\gamma_{3} ; y ; t\right) \\
& +\Omega_{\mathrm{S}}\left(\gamma_{1}+\gamma_{2}+\gamma_{3} ; y ; t\right)+2 \Omega_{\mathrm{S}}\left(\gamma_{1}+2 \gamma_{2}+\gamma_{3} ; y ; t\right)+\Omega_{\mathrm{S}}\left(\gamma_{1}+3 \gamma_{2}+\gamma_{3} ; y ; t\right) \\
& Q^{\prime}(1,1,1)=2\left(y^{-1}+y\right)^{2} \Omega_{\mathrm{S}}^{\prime}\left(\gamma_{1}^{\prime} ; y ; t\right) \Omega_{\mathrm{S}}^{\prime}\left(\gamma_{3}^{\prime} ; y ; t\right) \\
& +2 \Omega_{\mathrm{S}}^{\prime}\left(\gamma_{1}^{\prime}+\gamma_{3}^{\prime} ; y ; t\right)+\Omega_{\mathrm{S}}^{\prime}\left(\gamma_{1}^{\prime}+\gamma_{2}^{\prime}+\gamma_{3}^{\prime} ; y ; t\right) \\
& Q(1,2,2)=\frac{1}{2}\left(y^{-2}+1+y^{2}\right)\left(y^{-2}+4+y^{2}\right)\left\{\left(y^{-2}+1+y^{2}\right) \Omega_{\mathrm{S}}\left(\gamma_{3} ; y ; t\right)^{2}\right. \\
& \left.\left.-\left(y^{-2}-1+y^{2}\right) \Omega_{\mathrm{S}}\left(\gamma_{3} ; y^{2} ; t^{2}\right)-2\left(y^{-2}-1+y^{2}\right)\left(y^{-1}+y\right) \Omega_{\mathrm{S}}\left(2 \gamma_{3} ; y ; t\right)\right)\right\} \Omega_{\mathrm{S}}\left(\gamma_{1} ; y ; t\right) \\
& +\left(y^{-2}+1+y^{2}\right) \Omega_{\mathrm{S}}\left(\gamma_{3} ; y ; t\right)\left\{\Omega_{\mathrm{S}}\left(\gamma_{1}+\gamma_{3} ; y ; t\right)+2 \Omega_{\mathrm{S}}\left(\gamma_{1}+\gamma_{2}+\gamma_{3} ; y ; t\right)\right. \\
& \left.+\Omega_{\mathrm{S}}\left(\gamma_{1}+2 \gamma_{2}+\gamma_{3} ; y ; t\right)\right\}+\Omega_{\mathrm{S}}\left(\gamma_{1}+2 \gamma_{2}+2 \gamma_{3} ; y ; t\right) \\
& Q^{\prime}(1,2,2)=\frac{1}{2}\left(y^{-2}+1+y^{2}\right)\left(y^{-2}+4+y^{2}\right)\left\{\left(y^{-2}+1+y^{2}\right) \Omega_{\mathrm{S}}^{\prime}\left(\gamma_{3}^{\prime} ; y ; t\right)^{2}\right. \\
& \left.\left.-\left(y^{-2}-1+y^{2}\right) \Omega_{\mathrm{S}}^{\prime}\left(\gamma_{3}^{\prime} ; y^{2} ; t^{2}\right)-2\left(y^{-2}-1+y^{2}\right)\left(y^{-1}+y\right) \Omega_{\mathrm{S}}^{\prime}\left(2 \gamma_{3}^{\prime} ; y ; t\right)\right)\right\} \Omega_{\mathrm{S}}^{\prime}\left(\gamma_{1}^{\prime} ; y ; t\right) \\
& +\left(y^{-2}+1+y^{2}\right) \Omega_{\mathrm{S}}^{\prime}\left(\gamma_{3}^{\prime} ; y ; t\right)\left\{\Omega_{\mathrm{S}}^{\prime}\left(\gamma_{1}^{\prime}+\gamma_{3}^{\prime} ; y ; t\right)+2 \Omega_{\mathrm{S}}^{\prime}\left(\gamma_{1}^{\prime}+\gamma_{2}^{\prime}+\gamma_{3}^{\prime} ; y ; t\right)\right. \\
& \left.+\Omega_{\mathrm{S}}^{\prime}\left(\gamma_{1}^{\prime}+2 \gamma_{2}^{\prime}+\gamma_{3}^{\prime} ; y ; t\right)\right\}+\Omega_{\mathrm{S}}^{\prime}\left(\gamma_{1}^{\prime}+2 \gamma_{2}^{\prime}+2 \gamma_{3}^{\prime} ; y ; t\right) \\
& Q(1,2,3)=\frac{1}{6}\left(y^{-2}+4+y^{2}\right)\left[\left(y^{-2}+1+y^{2}\right)^{3} \Omega_{\mathrm{S}}\left(\gamma_{3} ; y ; t\right)^{3}\right. \\
& -3\left(y^{-2}-1+y^{2}\right)\left(y^{-2}+1+y^{2}\right)^{2} \Omega_{\mathrm{S}}\left(\gamma_{3} ; y ; t\right)\left\{\Omega_{\mathrm{S}}\left(\gamma_{3} ; y^{2} ; t^{2}\right)+2\left(y^{-1}+y\right) \Omega_{\mathrm{S}}\left(2 \gamma_{3} ; y ; t\right)\right\} \\
& \left.+2\left(y^{-6}+1+y^{6}\right)\left\{\Omega_{\mathrm{S}}\left(\gamma_{3} ; y^{3} ; t^{3}\right)+3\left(y^{-2}+1+y^{2}\right) \Omega_{\mathrm{S}}\left(3 \gamma_{3} ; y ; t\right)\right\}\right] \Omega_{\mathrm{S}}\left(\gamma_{1} ; y ; t\right) \\
& +\frac{1}{2}\left\{\left(y^{-2}+1+y^{2}\right)^{2} \Omega_{\mathrm{S}}\left(\gamma_{3} ; y ; t\right)^{2}-\left(y^{-4}+1+y^{4}\right) \Omega_{\mathrm{S}}\left(\gamma_{3} ; y^{2} ; t^{2}\right)\right. \\
& \left.-2\left(y^{-5}+y^{-3}+y^{-1}+y+y^{3}+y^{5}\right) \Omega_{\mathrm{S}}\left(2 \gamma_{3} ; y ; t\right)\right\} \\
& \times\left\{\Omega_{\mathrm{S}}\left(\gamma_{1}+\gamma_{3} ; y ; t\right)+2 \Omega_{\mathrm{S}}\left(\gamma_{1}+\gamma_{2}+\gamma_{3} ; y ; t\right)+\Omega_{\mathrm{S}}\left(\gamma_{1}+2 \gamma_{2}+\gamma_{3} ; y ; t\right)\right\} \\
& +\left(y^{-2}+1+y^{2}\right) \Omega_{\mathrm{S}}\left(\gamma_{3} ; y ; t\right) \Omega_{\mathrm{S}}\left(\gamma_{1}+2 \gamma_{2}+2 \gamma_{3} ; y ; t\right) \\
& +\Omega_{\mathrm{S}}\left(\gamma_{1}+2 \gamma_{2}+3 \gamma_{3} ; y ; t\right) \\
& Q^{\prime}(1,2,3)=\frac{1}{6}\left(y^{-2}+4+y^{2}\right)\left[\left(y^{-2}+1+y^{2}\right)^{3} \Omega_{\mathrm{S}}^{\prime}\left(\gamma_{3}^{\prime} ; y ; t\right)^{3}\right. \\
& -3\left(y^{-2}-1+y^{2}\right)\left(y^{-2}+1+y^{2}\right)^{2} \Omega_{\mathrm{S}}^{\prime}\left(\gamma_{3}^{\prime} ; y ; t\right)\left\{\Omega_{\mathrm{S}}^{\prime}\left(\gamma_{3}^{\prime} ; y^{2} ; t^{2}\right)+2\left(y^{-1}+y\right) \Omega_{\mathrm{S}}^{\prime}\left(2 \gamma_{3}^{\prime} ; y ; t\right)\right\} \\
& \left.+2\left(y^{-6}+1+y^{6}\right)\left\{\Omega_{\mathrm{S}}^{\prime}\left(\gamma_{3}^{\prime} ; y^{3} ; t^{3}\right)+3\left(y^{-2}+1+y^{2}\right) \Omega_{\mathrm{S}}^{\prime}\left(3 \gamma_{3}^{\prime} ; y ; t\right)\right\}\right] \Omega_{\mathrm{S}}^{\prime}\left(\gamma_{1}^{\prime} ; y ; t\right) \\
& +\frac{1}{2}\left\{\left(y^{-2}+1+y^{2}\right)^{2} \Omega_{\mathrm{S}}^{\prime}\left(\gamma_{3}^{\prime} ; y ; t\right)^{2}-\left(y^{-4}+1+y^{4}\right) \Omega_{\mathrm{S}}^{\prime}\left(\gamma_{3}^{\prime} ; y^{2} ; t^{2}\right)\right. \\
& \left.-2\left(y^{-5}+y^{-3}+y^{-1}+y+y^{3}+y^{5}\right) \Omega_{\mathrm{S}}^{\prime}\left(2 \gamma_{3}^{\prime} ; y ; t\right)\right\} \\
& \times\left\{\Omega_{\mathrm{S}}^{\prime}\left(\gamma_{1}^{\prime}+\gamma_{3}^{\prime} ; y ; t\right)+2 \Omega_{\mathrm{S}}^{\prime}\left(\gamma_{1}^{\prime}+\gamma_{2}^{\prime}+\gamma_{3}^{\prime} ; y ; t\right)+\Omega_{\mathrm{S}}^{\prime}\left(\gamma_{1}^{\prime}+2 \gamma_{2}^{\prime}+\gamma_{3}^{\prime} ; y ; t\right)\right\}
\end{aligned}
$$




$$
\begin{aligned}
& +\left(y^{-2}+1+y f^{2}\right) \Omega_{\mathrm{S}}^{\prime}\left(\gamma_{3}^{\prime} ; y ; t\right) \Omega_{\mathrm{S}}^{\prime}\left(\gamma_{1}^{\prime}+2 \gamma_{2}^{\prime}+2 \gamma_{3}^{\prime} ; y ; t\right) \\
& +\Omega_{\mathrm{S}}^{\prime}\left(\gamma_{1}^{\prime}+2 \gamma_{2}^{\prime}+3 \gamma_{3}^{\prime} ; y ; t\right) .
\end{aligned}
$$

Using (4.44) we see that these results are in agreement with (4.47).

We have checked similar agreement for many other examples.

\section{Acknowledgments}

We would like to thank Bernhard Keller, Gregory W. Moore, Andy Neitzke and Piljin Yi for inspiring discussions. Part of the reported results were obtained while J.M. was a postdoc of the Bethe Center for Theoretical Physics of Bonn University. This work was supported in part by the National Science Foundation under Grant No. PHYS-1066293 and the hospitality of the Aspen Center for Physics. The work of A.S. was supported in part by DAE project 12-R\&D-HRI-5.02-0303 and the J.C. Bose fellowship of DST, Govt. of India.

Open Access. This article is distributed under the terms of the Creative Commons Attribution License (CC-BY 4.0), which permits any use, distribution and reproduction in any medium, provided the original author(s) and source are credited.

\section{References}

[1] M.R. Douglas and G.W. Moore, D-branes, quivers and ALE instantons, hep-th/9603167 [INSPIRE].

[2] B. Fiol, The BPS spectrum of $N=2 \mathrm{SU}(N) S Y M$ and parton branes, hep-th/0012079 [INSPIRE].

[3] B. Fiol and M. Mariño, BPS states and algebras from quivers, JHEP 07 (2000) 031 [hep-th/0006189] [INSPIRE].

[4] M. Alim et al., BPS quivers and spectra of complete $N=2$ quantum field theories, Commun. Math. Phys. 323 (2013) 1185 [arXiv:1109.4941] [INSPIRE].

[5] M. Alim et al., $N=2$ quantum field theories and their BPS quivers, arXiv:1112.3984 [INSPIRE].

[6] S. Cecotti, The quiver approach to the BPS spectrum of a $4 d N=2$ gauge theory, arXiv: 1212.3431 [INSPIRE].

[7] D. Xie, BPS spectrum, wall crossing and quantum dilogarithm identity, arXiv:1211.7071 [INSPIRE].

[8] D. Galakhov, P. Longhi, T. Mainiero, G.W. Moore and A. Neitzke, Wild wall crossing and BPS giants, JHEP 11 (2013) 046 [arXiv:1305.5454] [INSPIRE].

[9] C. Cordova and A. Neitzke, Line defects, tropicalization and multi-centered quiver quantum mechanics, arXiv:1308.6829 [INSPIRE].

[10] W.-Y. Chuang, D.-E. Diaconescu, J. Manschot, G.W. Moore and Y. Soibelman, Geometric engineering of (framed) BPS states, arXiv:1301.3065 [INSPIRE].

[11] M. Cirafici, Line defects and (framed) BPS quivers, JHEP 11 (2013) 141 [arXiv:1307.7134] [INSPIRE]. 
[12] M.R. Douglas, B. Fiol and C. Romelsberger, Stability and BPS branes, JHEP 09 (2005) 006 [hep-th/0002037] [INSPIRE].

[13] F. Denef, Quantum quivers and Hall/hole halos, JHEP 10 (2002) 023 [hep-th/0206072] [INSPIRE].

[14] F. Denef and G.W. Moore, Split states, entropy enigmas, holes and halos, JHEP 11 (2011) 129 [hep-th/0702146] [INSPIRE].

[15] M. Aganagic and K. Schaeffer, Wall crossing, quivers and crystals, JHEP 10 (2012) 153 [arXiv: 1006.2113] [INSPIRE].

[16] A. King, Moduli of representations of finite dimensional algebras, Quart. J. Math. Oxford 45 (1994) 515.

[17] M. Reineke, The Harder-Narasimhan system in quantum groups and cohomology of quiver moduli, Invent. Math. 152 (2003) 349 [math.QA/0204059].

[18] J. de Boer, S. El-Showk, I. Messamah and D. Van den Bleeken, Quantizing $N=2$ multicenter solutions, JHEP 05 (2009) 002 [arXiv:0807.4556] [INSPIRE].

[19] J. Manschot, B. Pioline and A. Sen, A fixed point formula for the index of multi-centered $N=2$ black holes, JHEP 05 (2011) 057 [arXiv:1103.1887] [INSPIRE].

[20] H. Kim, J. Park, Z. Wang and P. Yi, Ab initio wall-crossing, JHEP 09 (2011) 079 [arXiv: 1107.0723] [INSPIRE].

[21] S. Lee and P. Yi, Framed BPS states, moduli dynamics and wall-crossing, JHEP 04 (2011) 098 [arXiv: 1102.1729] [INSPIRE].

[22] J. Manschot, B. Pioline and A. Sen, Wall crossing from Boltzmann black hole halos, JHEP 07 (2011) 059 [arXiv: 1011.1258] [INSPIRE].

[23] J. Manschot, B. Pioline and A. Sen, On the Coulomb and Higgs branch formulae for multi-centered black holes and quiver invariants, JHEP 05 (2013) 166 [arXiv:1302.5498] [INSPIRE].

[24] B. Pioline, Corfu lectures on wall-crossing, multi-centered black holes and quiver invariants, PoS (Corfu2012) 085 [arXiv: 1304.7159] [INSPIRE].

[25] A. Sen, Equivalence of three wall-crossing formulae, Commun. Num. Theor. Phys. 6 (2012) 601 [arXiv:1112.2515] [INSPIRE].

[26] I. Bena, M. Berkooz, J. de Boer, S. El-Showk and D. Van den Bleeken, Scaling BPS solutions and pure-Higgs states, JHEP 11 (2012) 171 [arXiv: 1205.5023] [INSPIRE].

[27] S.-J. Lee, Z.-L. Wang and P. Yi, Quiver invariants from intrinsic Higgs states, JHEP 07 (2012) 169 [arXiv:1205.6511] [inSPIRE].

[28] S.-J. Lee, Z.-L. Wang and P. Yi, BPS states, refined indices and quiver invariants, JHEP 10 (2012) 094 [arXiv: 1207.0821] [INSPIRE].

[29] J. Manschot, B. Pioline and A. Sen, From black holes to quivers, JHEP 11 (2012) 023 [arXiv: 1207.2230] [INSPIRE].

[30] I. Bena, C.-W. Wang and N.P. Warner, Mergers and typical black hole microstates, JHEP 11 (2006) 042 [hep-th/0608217] [INSPIRE].

[31] H. Derksen, J. Weyman and A. Zelevinsky, Quivers with potentials and their representations. I: mutations, Selecta Math. 14 (2008) 59 [arXiv: 0704.0649]. 
[32] M. Kontsevich and Y. Soibelman, Stability structures, motivic Donaldson-Thomas invariants and cluster transformations, arXiv:0811.2435 [INSPIRE].

[33] B. Keller and D. Yang, Derived equivalences from mutations of quivers with potential, Adv. Math. 226 (2011) 2118 [arXiv:0906.0761].

[34] I.N. Bernstein, I.M. Gelfand and V.A. Ponomarev, Coxeter functors, and Gabriel's theorem, Russ. Math. Surv. 28 (1973) 17 [Uspehi Mat. Nauk 28 (1973) 19]

[35] S. Fomin and A. Zelevinsky, Cluster algebras I: foundations, math.RT/0104151.

[36] B. Feng, A. Hanany, Y.-H. He and A.M. Uranga, Toric duality as Seiberg duality and brane diamonds, JHEP 12 (2001) 035 [hep-th/0109063] [INSPIRE].

[37] C.E. Beasley and M.R. Plesser, Toric duality is Seiberg duality, JHEP 12 (2001) 001 [hep-th/0109053] [INSPIRE].

[38] D. Berenstein and M.R. Douglas, Seiberg duality for quiver gauge theories, hep-th/0207027 [INSPIRE].

[39] B. Feng, A. Hanany, Y.H. He and A. Iqbal, Quiver theories, soliton spectra and Picard-Lefschetz transformations, JHEP 02 (2003) 056 [hep-th/0206152] [INSPIRE].

[40] S. Mukhopadhyay and K. Ray, Seiberg duality as derived equivalence for some quiver gauge theories, JHEP 02 (2004) 070 [hep-th/0309191] [INSPIRE].

[41] C.P. Herzog, Seiberg duality is an exceptional mutation, JHEP 08 (2004) 064 [hep-th/0405118] [INSPIRE].

[42] J. Vitoria, Mutations vs. Seiberg duality, J. Algebra 321 (2009) 816 [arXiv:0709.3939] [INSPIRE].

[43] F. Denef, Supergravity flows and D-brane stability, JHEP 08 (2000) 050 [hep-th/0005049] [INSPIRE].

[44] E. Andriyash, F. Denef, D.L. Jafferis and G.W. Moore, Bound state transformation walls, JHEP 03 (2012) 007 [arXiv: 1008.3555] [INSPIRE].

[45] D. Joyce and Y. Song, A theory of generalized Donaldson-Thomas invariants, Mem. Amer. Math. Soc. 217 (2012) iv+199 [arXiv:0810.5645] [INSPIRE].

[46] S. Fomin and A. Zelevinsky, Cluster algebras. IV: coefficients, Composit. Math. 143 (2007) 112 [math. RA/0602259].

[47] V. Fock and A. Goncharov, Cluster ensembles, quantization and the dilogarithm, math.AG/0311245 [INSPIRE].

[48] D. Labardini-Fragoso and A. Zelevinsky, Strongly primitive species with potentials I: mutations, arXiv:1306.3495.

[49] S. Alexandrov, D. Persson and B. Pioline, Wall-crossing, Rogers dilogarithm and the QK/HK correspondence, JHEP 12 (2011) 027 [arXiv:1110.0466] [INSPIRE].

[50] T. Dimofte and S. Gukov, Refined, motivic and quantum, Lett. Math. Phys. 91 (2010) 1 [arXiv: 0904.1420] [INSPIRE]. 